\title{
m-Polar Picture Fuzzy Ideal of a BCK Algebra
}

\author{
Shovan Dogra, Madhumangal Pal ${ }^{*}$ \\ Department of Applied Mathematics with Oceanology and Computer Programming, Vidyasagar University, Midnapore 721102, India
}

\author{
ARTICLE INFO \\ Article History \\ Received 17 Dec 2019 \\ Accepted 26 Mar 2020 \\ Keywords \\ $m$-polar picture fuzzy ideal \\ Homomorphism of $m$-polar picture \\ fuzzy ideal \\ $m$-polar picture fuzzy implicative \\ ideal \\ $m$-polar picture fuzzy commutative \\ ideal
}

\begin{abstract}
In this paper, the notions of $m$-polar picture fuzzy subalgebra (PFSA), $m$-polar picture fuzzy ideal (PFI) and $m$-polar picture fuzzy implicative ideal (PFII) of BCK algebra are introduced and some related basic results are presented. A relation between $m$ polar PFI and $m$-polar PFII is established. It is shown that an $m$-polar PFII of a BCK algebra is an $m$-polar PFI. But the converse of the proposition is not necessarily true. Converse is true only in implicative BCK algebra. The concept of $m$-polar picture fuzzy commutative ideal (PFCI) is also explored here and some related results are investigated.
\end{abstract}

(c) 2020 The Authors. Published by Atlantis Press SARL. This is an open access article distributed under the CC BY-NC 4.0 license (http://creativecommons.org/licenses/by-nc/4.0/).

\section{INTRODUCTION}

After the initiation of fuzzy set (FS) by Zadeh [1] in 1965, the notion of intuitionistic fuzzy set (IFS) was propounded by Atanassov [2] in 1986. IFS includes both the degree of membership (DMS) and the degree of non-membership (DNonMS), whereas fuzzy set includes only the DMS. The concept of BCI/BCK algebra was presented by Iseki and co-workers [3-5]. Merging the concepts of FS and BCK algebra, fuzzy BCK algebra was initiated by Xi [6]. In 1993, the idea of FS was connected with BCI algebra by Ahmad [7]. Later on a lot of works on BCK/BCI algebra and ideals in fuzzy set environment were done by several researchers [8-12]. Intuitionistic fuzzy subalgebra and intuitionistic fuzzy ideal (IFI) in BCK algebra were presented by Jun and Kim [13] in 2000 as an extension of FS concept in BCK algebra. As the time goes, BCK/BCI algebra and ideals were studied by Senapati et al. $[14,15]$ in context of intuitionistic in various directions. Bipolar fuzzy set (BFS) [16] is the generalization of FS which involves the degree of positive membership (DPMS) and the degree of negative membership (DNegMS) of an element. Bipolar fuzzy environment can be realized by an example. The serials broadcasted in Television have both good effect and bad effect on young generation. Good effect can be treated as positive effect and bad effect can be treated as negative effect. Extension work on BFSwas given by Chen [17] in the form of $m$-polar FS. In 2013, including the measure of neutral membership and generalizing the notion of IFS, the concept of picture fuzzy set (PFS) was initiated by Cuong [18]. After the initiation of PFS, different types of research works in context of PFS were performed by several researchers

\footnotetext{
*Corresponding author. Email: mmpalvu@gmail.com
}

[19-21]. In this paper, we introduce the concept of $m$-polar picture fuzzy subalgebra (PFSA), $m$-polar picture fuzzy ideal (PFI) and $m$-polar picture fuzzy implicative ideal (PFII), $m$-polar picture fuzzy commutative ideal (PFII) of BCK algebra and explore some results related to these. Also, we develop relationships of $m$-polar PFI with $m$-polar PFII and m-polar PFCI of BCK algebra.

\section{LIST OF ABBREVIATIONS}

FS - Fuzzy set

IFS - Intuitionistic fuzzy set

BFS - Bipolar fuzzy Set

PFS - Picture fuzzy set

DMS - Degree of membership

DNonMS - Degree of non-membership

DPMS - Degree of positive membership

DNegMS - Degree of negative membership

DNeuMS - Degree of neutral membership-

FI - Fuzzy ideal

IFI - Intuitionistic fuzzy ideal

PFSA - Picture fuzzy subalgebra

PFI - Picture fuzzy ideal 
PFII - Picture fuzzy implicative ideal

PFPII - Picture fuzzy positive implicative ideal

PFCI - Picture fuzzy commutative ideal

\section{PRELIMINARIES}

Here, we recapitulate some basic concepts of FS, IFS, BCK/BCI algebra, FI, IFI, BFS, $m$-polar FS and PFS. We define $m$-polar PFS, some basic operations on $m$-polar PFSs, $(\theta, \phi, \psi)$-cut of $m$-polar PFS, image and inverse of $m$-polar PFS.

Definition 1. Let $A$ be the set of universe. Then a FS [1] $P$ over $A$ is defined as $P=\left\{\left(a, \mu_{P}(a)\right): a \in A\right\}$, where $\mu_{P}: A \rightarrow[0,1]$. Here, $\mu_{P}(a)$ is DMS of $a$ in $P$.

The DNonMS was missing in FS. Including this type of uncertainty, Atanassov defined IFS in 1986.

Definition 2. Let $A$ be the set of universe. An IFS [2] $P$ over $A$ is defined as $P=\left\{\left(a, \mu_{P}(a), v_{P}(a)\right): a \in A\right\}$, where $\mu_{P}(a) \in[0,1]$ is the DMS of $a$ in $P$ and $v_{P}(a) \in[0,1]$ is the DNonMS of $a$ in $P$ with the condition $0 \leqslant \mu_{P}(a)+v_{P}(a) \leqslant 1$ for all $a \in A$.

Here, $s_{P}(a)=1-\left(\mu_{P}(a)+v_{P}(a)\right)$ is the measure of suspicion of $a$ in $P$, which excludes the DMS and the DNonMS.

Iseki introduced a special type of algebra namely BCI algebra in 1980.

Definition 3. An algebra $(A, \diamond, 0)$ is said to be BCI algebra [4] if for any $a, b, c \in A$, the below stated conditions are meet.

$$
\begin{aligned}
\text { i. } & {[(a \diamond b) \diamond(a \diamond c)] \diamond(c \diamond b)=0 } \\
\text { ii. } & {[a \diamond(a \diamond b)] \diamond b=0 } \\
\text { iii. } & a \diamond a=0 \\
\text { iv. } & a \diamond b=0 \text { and } b \diamond a=0 \Rightarrow a=b
\end{aligned}
$$

A BCI algebra with the condition $0 \diamond a=0$ for all $a \in A$ is called BCK algebra.

A relation " $\leqslant$ " on $A$ is defined as $a \leqslant b$ iff $a \diamond b=0$.

Proposition 1. In a BCK algebra $(A, \diamond, 0)$ the followings hold.

$$
\begin{aligned}
\text { i. } & 0 \diamond a=0 \\
\text { ii. } & a \diamond 0=a \\
\text { iii. } & a \diamond(a \diamond b) \leqslant b \\
\text { iv. } & a \diamond b \leqslant a \\
\text { v. } & (a \diamond b) \diamond c=(a \diamond c) \diamond b \\
\text { vi. } & (a \diamond(a \diamond(a \diamond b)))=a \diamond b \text { for all } a, b, c \in A
\end{aligned}
$$

Definition 4. Let $(A, \diamond, 0)$ be a BCK algebra and $P=\left(\mu_{P}, \eta_{P}, v_{P}\right)$ be a FS in $A$. Then $P$ is said to be FI [6] of $A$ if

$$
\begin{array}{cl}
\text { i. } & \mu_{P}(0) \geqslant \mu_{P}(a) \\
\text { ii. } & \mu_{P}(a) \geqslant \mu_{P}(a \diamond b) \wedge \mu_{P}(b) \text { for all } a, b \in A \text { and for } l=1,2, \ldots, m
\end{array}
$$

Definition 5. Let $(A, \diamond, 0)$ be a BCK algebra and $P=\left(\mu_{P}, \eta_{P}, v_{P}\right)$ be an IFS in $A$. Then $P$ is said to IFI [13] of $A$ if

i. $\quad \mu_{P}(0) \geqslant \mu_{P}(a)$ and $v_{P}(0) \leqslant v_{P}(a)$

ii. $\quad \mu_{P}(a) \geqslant \mu_{P}(a \diamond b) \wedge \mu_{P}(b)$ and $v_{P}(a) \leqslant v_{P}(a \diamond b) \vee v_{P}(b)$ for all $a, b \in A$

Definition 6. A BFS [16] $P$ is defined as $P=\left(a, \mu_{P}(a), v_{P}(a)\right): a \in$ $A$, where $\mu_{P}(a) \in(0,1]$ measures how much a particular property is satisfied by an element and $v_{P}(a) \in[-1,0)$ measures how much its anti property is satisfied by that element. DMS 0 means the element has no relevancy to the property.

Definition 7. An $m$-polar FS [17] $P$ over the set of universe $A$ is an object of the form $P=\left\{\left(a, \mu_{P}(a)\right): a \in A\right\}$, where $\mu_{P}$ : $A \rightarrow[0,1]^{m}$ ( $m$ is a natural number). Here, $[0,1]^{m}$ is the poset with respect to partial order relation " $\leqslant$ " which is defined as: $a \leqslant b$ iff $p_{l}(a) \leqslant p_{l}(b)$ for $l=1,2, \ldots, m$; where $p_{l}:[0,1]^{m} \rightarrow[0,1]$ is called $l$-th projection mapping.

Including more possible types of uncertainity, Cuong defined PFS in 2013 generalizing the concepts of FS and IFS.

Definition 8. Let $A$ be the set of universe. Then a PFS [18] $P$ over the universe $A$ is defined as $P=\left\{\left(a, \mu_{P}(a), \eta_{P}(a), v_{P}(a)\right): a \in A\right\}$, where $\mu_{P}(a) \in[0,1]$ is the DPMS of $a$ in $P, \eta_{P}(a) \in[0,1]$ is the degree of neutral membership (DNeuMS) of $a$ in $P$ and $v_{P}(a) \in$ $[0,1]$ is the DNegMS of $a$ in $P$ with the condition $0 \leqslant \mu_{P}(a)+$ $\eta_{P}(a)+v_{P}(a) \leqslant 1$ for all $a \in A$. For all $a \in A, 1-\left(\mu_{P}(a)+\right.$ $\left.\eta_{P}(a)+v_{P}(a)\right)$ is the measure of denial membership $a$ in $P$. Sometimes, $\left(\mu_{P}(a), \eta_{P}(a), v_{P}(a)\right)$ is called picture fuzzy value for $a \in A$.

Motivated by this definition, below we define $m$-polar PFS.

Definition 9. An $m$-polar PFS $P$ over the set of universe $A$ is an object of the form $P=\left\{\left(a, \mu_{P}(a), \eta_{P}(a), v_{P}(a)\right): a \in A\right\}$, where $\mu_{P}: A \rightarrow[0,1]^{m}, \eta_{P}: A \rightarrow[0,1]^{m}$ and $v_{P}: A \rightarrow[0,1]^{m}(m$ is a natural number) with the condition $0 \leqslant p_{l} \circ \mu_{P}(a)+p_{l} \circ \eta_{P}(a)+$ $p_{l} \circ v_{P}(a) \leqslant 1$ for all $a \in A$ and for $l=1,2, \ldots, m$. For $a \in A$, each of $\mu_{P}(a), \eta_{P}(a)$ and $v_{P}(a)$ is an $m$-tuple fuzzy value. Here, $p_{l} \circ \mu_{P}(a)$, $p_{l} \circ \eta_{P}(a)$ and $p_{l} \circ v_{P}(a)$ represent $l$-th components of $\mu_{P}(a), \eta_{P}(a)$ and $v_{P}(a)$ respectively for $l=1,2, \ldots, m$.

The basic operations on $m$-polar PFSs consisting of equality, union and intersection are defined below.

Definition 10. Let $P=\left\{\left(a, \mu_{P}(a), \eta_{P}(a), v_{P}(a)\right): a \in A\right\}$ and $Q=$ $\left\{\left(a, \mu_{Q}(a), \eta_{Q}(a), v_{Q}(a)\right): a \in A\right\}$ be two $m$-polar PFSs over the universe $A$. Then

i. $\quad P \subseteq Q$ iff $p_{l} \circ \mu_{P}(a) \leqslant p_{l} \circ \mu_{Q}(a), p_{l} \circ \eta_{P}(a) \leqslant p_{l} \circ \eta_{Q}(a)$ and $p_{l} \circ v_{P}(a) \geqslant p_{l} \circ v_{Q}(a)$ for all $a \in A$ and for $l=1,2, \ldots, m$.

ii. $\quad P=Q$ iff $p_{l} \circ \mu_{P}(a)=p_{l} \circ \mu_{Q}(a), p_{l} \circ \eta_{P}(a)=p_{l} \circ \eta_{Q}(a)$ and $p_{l} \circ v_{P}(a)=p_{l} \circ v_{Q}(a)$ for all $a \in A$ and for $l=1,2, \ldots, m$.

iii. $\quad p_{l} \circ(P \cup Q)=\left\{\left(a, \max \left(p_{l} \circ \mu_{P}(a), p_{l} \circ \mu_{Q}(a)\right), \min \left(p_{l} \circ \eta_{P}(a), p_{l} \circ\right.\right.\right.$ $\left.\left.\left.\eta_{Q}(a)\right), \min \left(p_{l} \circ v_{P}(a), p_{l} \circ v_{Q}(a)\right)\right): a \in A\right\}$ for $l=1,2, \ldots, m$.

iv. $p_{l} \circ(P \cap Q)=\left\{\left(a, \min \left(p_{l} \circ \mu_{P}(a), p_{l} \circ \mu_{Q}(a)\right), \min \left(p_{l} \circ \eta_{P}(a), p_{l} \circ\right.\right.\right.$ $\left.\left.\left.\eta_{Q}(a)\right), \max \left(p_{l} \circ v_{P}(a), p_{l} \circ v_{Q}(a)\right)\right): a \in A\right\}$ for $l=1,2, \ldots, m$.

Definition 11. Let $P=\left\{\left(a, \mu_{P}, \eta_{P}, v_{P}\right): a \in A\right\}$ be an m-polar PFS over the universe $A$. Then $(\theta, \phi, \psi)$-cut of $P$ is the crisp set in 
$A$ denoted by $C_{\theta, \phi, \psi}(P)$ and is defined as $C_{\theta, \phi, \psi}(P)=\{a \in A$ : $p_{l} \circ \mu_{P}(a) \geqslant p_{l} \circ \theta, p_{l} \circ \eta_{P}(a) \geqslant p_{l} \circ \phi, p_{l} \circ v_{P}(a) \leqslant p_{l} \circ \psi$ for $l=1,2, \ldots, m\}$, where $p_{l} \circ \theta \in[0,1], p_{l} \circ \phi \in[0,1], p_{l} \circ \psi \in[0,1]$ with the condition $0 \leqslant p_{l} \circ \theta+p_{l} \circ \phi+p_{l} \circ \psi \leqslant 1$ for $l=1,2, \ldots, m$. The mentionable fact is that each of $\theta, \phi$ and $\psi$ is an $m$-polar fuzzy value. Here, $p_{l} \circ \theta, p_{l} \circ \phi$ and $p_{l} \circ \psi$ represent $l$-th components of the $m$-polar fuzzy values $\theta, \phi$ and $\psi$ for $l=1,2, \ldots, m$

Definition 12. Let $A_{1}$ and $A_{2}$ be two sets of universe. Let $h: A_{1} \rightarrow A_{2}$ be a surjective mapping and $P=$ $\left\{\left(a_{1}, \mu_{P}\left(a_{1}\right), \eta_{P}\left(a_{1}\right), v_{P}\left(a_{1}\right)\right): a_{1} \in A_{1}\right\}$ be an $m$-polar PFS in $A_{1}$. Then the image of $P$ under the map $h$ is the $m$-polar PFS $h(P)=\left\{\left(a_{2}, \mu_{h(P)}\left(a_{2}\right), \eta_{h(P)}\left(a_{2}\right), v_{h(P)}\left(a_{2}\right)\right): a_{2} \in A_{2}\right\}$, where $p_{l}$ 。 $\mu_{h(P)}\left(a_{2}\right)=\underset{a_{1} \in h^{-1}\left(a_{2}\right)}{\vee} p_{l} \circ \mu_{P}\left(a_{1}\right), p_{l} \circ \eta_{h(P)}\left(a_{2}\right)=\underset{a_{1} \in h^{-1}\left(a_{2}\right)}{\wedge} p_{l} \circ \eta_{P}\left(a_{1}\right)$ and $p_{l} \circ v_{h(P)}\left(a_{2}\right) \stackrel{\wedge}{=} \wedge_{a_{1} \in h^{-1}\left(a_{2}\right)} p_{l} \circ v_{P}\left(a_{1}\right)$ for all $a_{2} \in A_{2}$ and for $l=1,2, \ldots, m$.

Definition 13. Let $A_{1}$ and $A_{2}$ be two sets of universe. Let $h$ : $A_{1} \rightarrow A_{2}$ be a mapping and $Q=\left\{\left(a_{2}, \mu_{Q}\left(a_{2}\right), \eta_{Q}\left(a_{2}\right), v_{Q}\left(a_{2}\right)\right)\right.$ : $\left.a_{2} \in A_{2}\right\}$ be an $m$-polar PFS in $A_{2}$. Then the inverse image of $Q$ under the map $h$ is the $m$-polar PFS $h^{-1}(Q)=$ $\left\{\left(a_{1}, \mu_{h^{-1}(Q)}\left(a_{1}\right), \eta_{h^{-1}(Q)}\left(a_{1}\right), v_{h^{-1}(Q)}\left(a_{1}\right)\right): a_{1} \in A_{1}\right\}$, where $p_{l}$ 。 $\mu_{h^{-1}(Q)}\left(a_{1}\right)=p_{l} \circ \mu_{Q}\left(h\left(a_{1}\right)\right), p_{l} \circ \eta_{h^{-1}(Q)}\left(a_{1}\right)=p_{l} \circ \eta_{Q}\left(h\left(a_{1}\right)\right)$ and $p_{l} \circ v_{h^{-1}(Q)}\left(a_{1}\right)=p_{l} \circ v_{Q}\left(h\left(a_{1}\right)\right)$ for all $a_{1} \in A_{1}$ and for $l=1,2, . ., m$.

Definition 14. Let $P=\left\{\left(a_{1}, \mu_{P}\left(a_{1}\right), \eta_{P}\left(a_{1}\right), v_{P}\left(a_{1}\right)\right): a_{1} \in A_{1}\right\}$ and $Q=\left\{\left(a_{2}, \mu_{Q}\left(a_{2}\right), \eta_{Q}\left(a_{2}\right), v_{Q}\left(a_{2}\right)\right): a_{2} \in A_{2}\right\}$ be two $m$ polar PFSs over the sets of universe $A_{1}$ and $A_{2}$ respectively. Then the Cartesian product of $P$ and $Q$ is the $m$-polar PFS $P \times Q=$ $\left\{\left((a, b), \mu_{P \times Q}((a, b)), \eta_{P \times Q}((a, b)), v_{P \times Q}((a, b))\right):(a, b) \in A_{1} \times A_{2}\right\}$, where $p_{l} \circ \mu_{P \times Q}((a, b))=p_{l} \circ \mu_{P}(a) \wedge p_{l} \circ \mu_{Q}(b), p_{l} \circ \eta_{P \times Q}((a, b))=$ $p_{l} \circ \eta_{P}(a) \wedge p_{l} \circ \eta_{Q}(b)$ and $p_{l} \circ v_{P \times Q}((a, b))=p_{l} \circ v_{P}(a) \vee p_{l} \circ v_{Q}(b)$ for all $(a, b) \in A_{1} \times A_{2}$ and for $l=1,2, \ldots, m$.

\section{4. m-POLAR PFI}

Let us first define $m$-polar PFSA of a BCK algebra.

Definition 15. Let $(A, \diamond, 0)$ be a BCK algebra and $P=\left(\mu_{P}, \eta_{P}, v_{P}\right)$ be an $m$-polar PFS in $A$. Then $P$ is said to be $m$-polar PFSA of $A$ if $p_{l} \circ \mu_{P}(a \diamond b) \geqslant p_{l} \circ \mu_{P}(a) \wedge p_{l} \circ \mu_{P}(b), p_{l} \circ \eta_{P}(a \diamond b) \geqslant p_{l} \circ \eta_{P}(a) \wedge$ $p_{l} \circ \eta_{P}(b)$ and $p_{l} \circ v_{P}(a \diamond b) \leqslant p_{l} \circ v_{P}(a) \vee p_{l} \circ v_{P}(b)$ for all $a, b \in A$ and for $l=1,2, \ldots, m$.

Example 1. Consider a BCK algebra $(A, \diamond, 0)$ defined in the following tabular form:

\begin{tabular}{lllll}
\hline$\diamond$ & 0 & $p$ & $q$ & $r$ \\
0 & 0 & 0 & 0 & 0 \\
$p$ & $p$ & 0 & 0 & $p$ \\
$q$ & $q$ & $p$ & 0 & $q$ \\
$r$ & $r$ & $r$ & $r$ & 0 \\
\hline
\end{tabular}

Now, let us consider a 3-polar PFS $P$ as follows:

$$
\mu_{P}(a)= \begin{cases}(0.25,0.35,0.4), & \text { if } a=0 \\ (0.15,0.25,0.35), & \text { if } a=p \\ (0.1,0.15,0.25), & \text { if } a=q \\ (0.15,0.25,0.4), & \text { if } a=r\end{cases}
$$

$$
\eta_{P}(a)= \begin{cases}(0.2,0.3,0.4), & \text { if } a=0 \\ (0.1,0.2,0.3), & \text { if } a=p \\ (0.05,0.1,0.2), & \text { if } a=q \\ (0.1,0.2,0.35), & \text { if } a=r\end{cases}
$$

and

$$
v_{P}(a)= \begin{cases}(0.1,0.15,0.2), & \text { if } a=0 \\ (0.15,0.2,0.3), & \text { if } a=p \\ (0.2,0.3,0.4), & \text { if } a=q \\ (0.15,0.2,0.25), & \text { if } a=r\end{cases}
$$

It is easy to show that $P$ is a 3-polar PFSA of $A$.

Proposition 2. Let $P=\left(\mu_{P}, \eta_{P}, v_{P}\right)$ be an m-polar PFSA of a BCK algebra $A$. Then $p_{l} \circ \mu_{P}(0) \geqslant p_{l} \circ \mu_{P}(a), p_{l} \circ \eta_{P}(0) \geqslant p_{l} \circ \eta_{P}(a)$ and $p_{l} \circ v_{P}(0) \leqslant p_{l} \circ v_{P}(a)$ for all $a \in A$ and for $l=1,2, \ldots, m$.

Proof. It is observed that

$$
\begin{aligned}
p_{l} \circ \mu_{P}(0)= & p_{l} \circ \mu_{P}(a \diamond a) \\
\geqslant & p_{l} \circ \mu_{P}(a) \wedge p_{l} \circ \mu_{P}(a) \\
& \quad[\text { because } P \text { is an } m \text {-polar PFSA of } A] \\
= & p_{l} \circ \mu_{P}(a), \\
p_{l} \circ \eta_{P}(0)= & p_{l} \circ \eta_{P}(a \diamond a) \\
\geqslant & p_{l} \circ \eta_{P}(a) \wedge p_{l} \circ \eta_{P}(a) \\
& {[\text { because } P \text { is an } m \text {-polar PFSA of } A] } \\
= & p_{l} \circ \eta_{P}(a)
\end{aligned}
$$

$$
\text { and } \begin{aligned}
p_{l} \circ v_{P}(0) & =p_{l} \circ v_{P}(a \diamond a) \\
& \leqslant p_{l} \circ v_{P}(a) \vee p_{l} \circ v_{P}(a)
\end{aligned}
$$

[because $P$ is an $m$-polar PFSA of $A$ ]

$$
=p_{l} \circ v_{P}(a) \text { for all } a \in A
$$

and for $l=1,2, \ldots, m$.

Thus, $p_{l} \circ \mu_{P}(0) \geqslant p_{l} \circ \mu_{P}(a), p_{l} \circ \eta_{P}(0) \geqslant p_{l} \circ \eta_{P}(a)$ and $p_{l} \circ v_{P}(0) \leqslant$ $p_{l} \circ v_{P}(a)$ for all $a \in A$ and for $l=1,2, \ldots, m$.

Now, let us define $m$-polar PFI of a BCK algebra.

Definition 16. Let $(A, \diamond, 0)$ be a BCK algebra and $P=\left(\mu_{P}, \eta_{P}, v_{P}\right)$ be an $m$-polar PFS in $A$. Then $P$ is said to $m$-polar PFI of $A$ if

i. $\quad p_{l} \circ \mu_{P}(0) \geqslant p_{l} \circ \mu_{P}(a), p_{l} \circ \eta_{P}(0) \geqslant p_{l} \circ \eta_{P}(a)$ and $p_{l} \circ v_{P}(0) \leqslant$ $p_{l} \circ v_{P}(a)$

ii. $\quad p_{l} \circ \mu_{P}(a) \geqslant p_{l} \circ \mu_{P}(a \diamond b) \wedge p_{l} \circ \mu_{P}(b), p_{l} \circ \eta_{P}(a) \geqslant p_{l} \circ \eta_{P}(a \diamond b) \wedge$ $p_{l} \circ \eta_{P}(b)$ and $p_{l} \circ v_{P}(a) \leqslant p_{l} \circ v_{P}(a \diamond b) \vee p_{l} \circ v_{P}(b)$ for all $a, b \in A$ and for $l=1,2, \ldots, m$

Now, we are going to investigate some important results on $m$-polar PFI of a BCK algebra.

Proposition 3. Let $P=\left(\mu_{P}, \eta_{P}, v_{P}\right)$ be an m-polar PFI of a BCK algebra $(A, \diamond, 0)$. Then $p_{l} \circ \mu_{P}(a) \geqslant p_{l} \circ \mu_{P}(b), p_{l} \circ \eta_{P}(a) \geqslant p_{l} \circ \eta_{P}(b)$ and $p_{l} \circ v_{P}(a) \leqslant p_{l} \circ v_{P}(b)$ for $a, b \in A$ with $a \leqslant b$ and for $l=$ $1,2, \ldots, m$. 
Proof. Let $a, b \in A$ such that $a \leqslant b$. Then $a \diamond b=0$.

Now, $p_{l} \circ \mu_{P}(a)$

$\geqslant p_{l} \circ \mu_{P}(a \diamond b) \wedge p_{l} \circ \mu_{P}(b)$ [as $P$ is an $m$-polar PFI of $A$ ]

$=p_{l} \circ \mu_{P}(0) \wedge p_{l} \circ \mu_{P}(b)$

$=p_{l} \circ \mu_{P}(b)$ [as $P$ is an $m$-polar PFI of $A$ ],

$p_{l} \circ \eta_{P}(a)$

$\geqslant p_{l} \circ \eta_{P}(a \diamond b) \wedge p_{l} \circ \eta_{P}(b)$ [as $P$ is an $m$-polar PFI of $A$ ]

$=p_{l} \circ \eta_{P}(0) \wedge p_{l} \circ \eta_{P}(b)$

$=p_{l} \circ \eta_{P}(b)[$ as $P$ is an $m$-polar PFI of $A$ ]

and $p_{l} \circ v_{P}(a)$

$\leqslant p_{l} \circ v_{P}(a \diamond b) \vee p_{l} \circ v_{P}(b)$ [as $P$ is an $m$-polar PFI of $A$ ]

$=p_{l} \circ v_{P}(0) \vee p_{l} \circ v_{P}(b)$

$=p_{l} \circ v_{P}(b)$ [as $P$ is an $m$-polar PFI of $A$ ]

for $l=1,2, \ldots, m$.

Thus, $p_{l} \circ \mu_{P}(a) \geqslant p_{l} \circ \mu_{P}(b), p_{l} \circ \eta_{P}(a) \geqslant p_{l} \circ \eta_{P}(b)$ and $p_{l} \circ v_{P}(a) \leqslant$ $p_{l} \circ v_{P}(b)$ for $a, b \in A$ with $a \leqslant b$ and for $l=1,2, \ldots, m$.

Proposition 4. Let $(A, \diamond, 0)$ be a $B C K$ algebra and $P=\left(\mu_{P}, \eta_{P}, v_{P}\right)$ be an m-polar PFI of $A$. Then $p_{l} \circ \mu_{P}(a) \geqslant p_{l} \circ \mu_{P}(b) \wedge p_{l} \circ \mu_{P}(c)$, $p_{l} \circ \eta_{P}(a) \geqslant p_{l} \circ \eta_{P}(b) \wedge p_{l} \circ \eta_{P}(c)$ and $p_{l} \circ v_{P}(a) \leqslant p_{l} \circ v_{P}(b) \vee p_{l} \circ v_{P}(c)$ for $a, b, c \in A$ with $a \diamond b \leqslant c$.

Proof. Let $a, b, c \in A$ with $a \diamond b \leqslant c$. Then $(a \diamond b) \diamond c=0$.

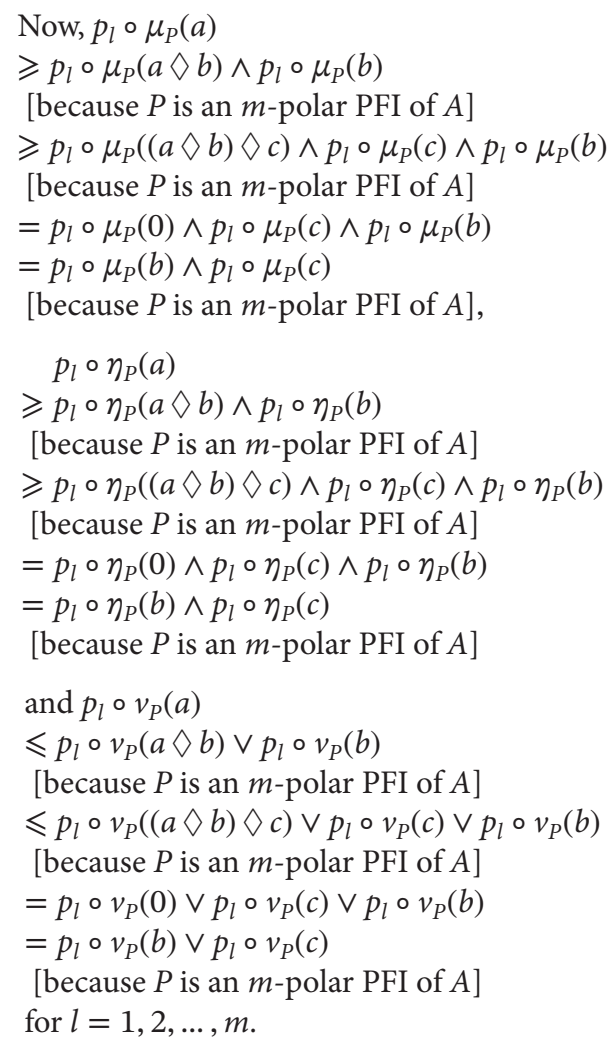

Thus, it is obtained that $p_{l} \circ \mu_{P}(a) \geqslant p_{l} \circ \mu_{P}(b) \wedge p_{l} \circ \mu_{P}(c), p_{l} \circ \eta_{P}(a) \geqslant$ $p_{l} \circ \eta_{P}(b) \wedge p_{l} \circ \eta_{P}(c)$ and $p_{l} \circ v_{P}(a) \leqslant p_{l} \circ v_{P}(b) \vee p_{l} \circ v_{P}(c)$ for $a, b, c \in A$ with $a \diamond b \leqslant c$.

Proposition 5. Every m-polar PFI of a BCK algebra is an m-polar PFSA.

Proof. Let $(A, \diamond, 0)$ be a BCK algebra and $A$ is an $m$-PFI of $A$. Since $P$ is an $m$-polar PFI, therefore,

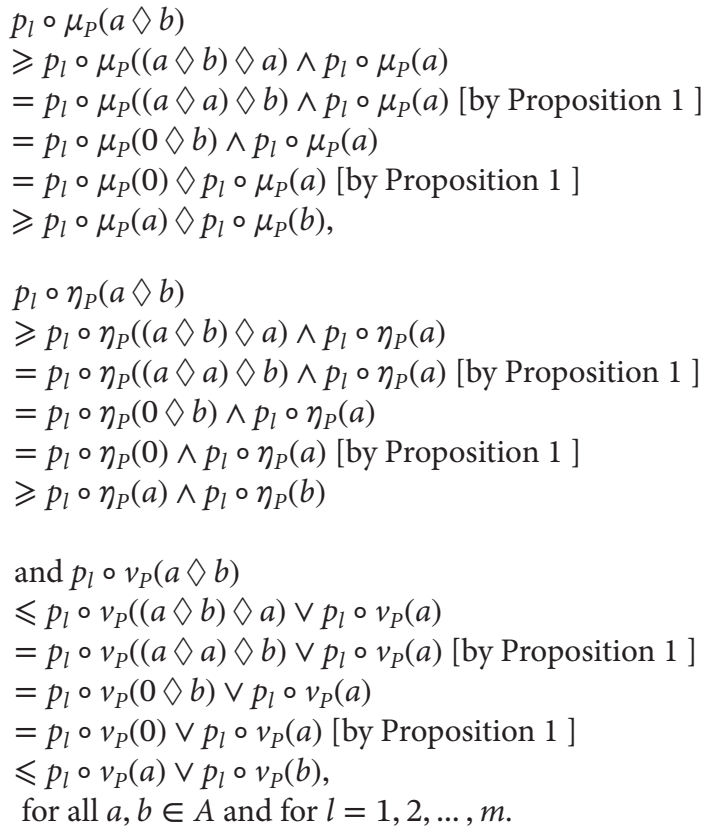

Hence, $P$ is an $m$-polar PFSA of $A$.

But, the converse of the above proposition is not true in general which is shown in following example. Proposition 6 states under which condition an $m$-polar PFSA is an $m$-polar PFI.

Example 2. Let us suppose the BCK algebra given in Example 1 and a 3-polar PFS $P$ as follows:

$$
\begin{gathered}
\mu_{P}(a)= \begin{cases}(0.2,0.3,0.4), & \text { if } a=0, q \\
(0.1,0.2,0.3), & \text { if } a=p, r\end{cases} \\
\eta_{P}(a)= \begin{cases}(0.25,0.35,0.45), & \text { if } a=0, q \\
(0.15,0.25,0.3), & \text { if } a=p, r\end{cases}
\end{gathered}
$$

and

$$
v_{P}(a)= \begin{cases}(0.3,0.2,0.1), & \text { if } a=0, q \\ (0.4,0.3,0.2), & \text { if } a=p, r\end{cases}
$$

Here, $(0.1,0.2,0.3)=\mu_{P}(p) \nsucceq \mu_{P}(p \diamond q) \wedge \mu_{P}(q)=(0.2,0.3,0.4)$, $(0.15,0.25,0.3)=\eta_{P}(p) \ngtr \eta_{P}(p \diamond q) \wedge \eta_{P}(q)=(0.25,0.35,0.45)$ and $(0.4,0.3,0.2)=v_{P}(p) \not \leq v_{P}(p \diamond q) \vee v_{P}(q)=(0.3,0.2,0.1)$. So, $P$ is not a 3-polar PFI of $A$ although it is a 3-polar PFSA.

Proposition 6. Let $P=\left(\mu_{P}, \eta_{P}, v_{P}\right)$ be an $m$-polar PFSA of a BCK algebra $(A, \nabla, 0)$. Then $P$ is an $m$-polar PFI of $A$ if for all $a, b, c \in A$, $a \diamond b \leqslant c \Rightarrow p_{l} \circ \mu_{P}(a) \geqslant p_{l} \circ \mu_{P}(b) \wedge p_{l} \circ \mu_{P}(c), p_{l} \circ \eta_{P}(a) \geqslant$ $p_{l} \circ \eta_{P}(b) \wedge p_{l} \circ \eta_{P}(c)$ and $p_{l} \circ v_{P}(a) \leqslant p_{l} \circ v_{P}(b) \vee p_{l} \circ v_{P}(c)$ for $l=1,2, \ldots, m$.

Proof. By given conditions, for all $a, b, c \in A, a \diamond b \leqslant c \Rightarrow p_{l}$ 。 $\mu_{P}(a) \geqslant p_{l} \circ \mu_{P}(b) \wedge p_{l} \circ \mu_{P}(c), p_{l} \circ \eta_{P}(a) \geqslant p_{l} \circ \eta_{P}(b) \wedge p_{l} \circ \eta_{P}(c)$ and $p_{l} \circ v_{P}(a) \leqslant p_{l} \circ v_{P}(b) \vee p_{l} \circ v_{P}(c)$. Since $A$ is a BCK algebra therefore by Proposition $1, a \diamond(a \diamond b) \leqslant b$. So, it is obtained that

$$
\begin{aligned}
p_{l} \circ \mu_{P}(a) \geqslant & p_{l} \circ \mu_{P}(a \diamond b) \wedge p_{l} \circ \mu_{P}(b) \\
p_{l} \circ \eta_{P}(a) \geqslant & p_{l} \circ \mu_{P}(a \diamond b) \wedge p_{l} \circ \mu_{P}(b) \\
\text { and } p_{l} \circ v_{P}(a) \leqslant & p_{l} \circ v_{P}(a \diamond b) \vee p_{l} \circ v_{P}(b) \\
& \text { for } l=1,2, \ldots, m
\end{aligned}
$$

Thus, $P$ is an $m$-polar PFI of $A$. 
Proposition 7. Let $(A, \nabla, 0)$ a $B C K$ algebra and $P=\left(\mu_{p}, \eta_{P}, v_{P}\right)$, $Q=\left(\mu_{Q}, \eta_{Q}, v_{Q}\right)$ be two m-polar PFIs of $A$. Then $P \cap Q$ is an m-polar PFI of $A$.

Proof. Let $P \cap Q=R=\left(\mu_{R}, \eta_{R}, v_{R}\right)$. Then $p_{l} \circ \mu_{R}(a)=p_{l} \circ \mu_{P}(a) \wedge$ $p_{l} \circ \mu_{Q}(a), p_{l} \circ \eta_{R}(a)=p_{l} \circ \eta_{P}(a) \wedge p_{l} \circ \eta_{Q}(a)$ and $p_{l} \circ v_{R}(a)=$ $p_{l} \circ v_{P}(a) \vee p_{l} \circ v_{Q}(a), \forall a \in A$ and for $l=1,2, \ldots, m$.

$$
\begin{aligned}
& \text { Now, } p_{l} \circ \mu_{R}(0) \\
& =p_{l} \circ \mu_{P}(0) \wedge p_{l} \circ \mu_{Q}(0) \\
& \geqslant p_{l} \circ \mu_{P}(a) \wedge p_{l} \circ \mu_{Q}(a) \\
& {[\text { as } P, Q \text { are } m \text {-polar PFIs of } A \text { ] }} \\
& =p_{l} \circ \mu_{R}(a) \\
& \quad p_{l} \circ \eta_{R}(0) \\
& =p_{l} \circ \eta_{P}(0) \wedge p_{l} \circ \eta_{Q}(0) \\
& \geqslant p_{l} \circ \eta_{P}(a) \wedge p_{l} \circ \eta_{Q}(a) \\
& {[\text { as } P, Q \text { are } m \text {-polar PFIs of } A \text { ] }} \\
& =p_{l} \circ \eta_{R}(a)
\end{aligned}
$$

$$
\begin{aligned}
& \text { and } p_{l} \circ v_{R}(0) \\
& =p_{l} \circ v_{P}(0) \vee p_{l} \circ v_{Q}(0) \\
& \leqslant p_{l} \circ v_{P}(a) \vee p_{l} \circ v_{Q}(a) \\
& {[\text { as } P, Q \text { are } m \text {-polar PFIs of } A]} \\
& =p_{l} \circ v_{R}(a), \forall a \in A \text { and } l=1,2, \ldots, m \text {. }
\end{aligned}
$$

$$
\begin{aligned}
& \text { Also, } p_{l} \circ \mu_{R}(a) \\
& =p_{l} \circ \mu_{P}(a) \wedge p_{l} \circ \mu_{Q}(a) \\
& \geqslant\left(p_{l} \circ \mu_{P}(a \diamond b) \wedge p_{l} \circ \mu_{P}(b)\right) \wedge\left(p_{l} \circ \mu_{Q}(a \diamond b) \wedge p_{l} \circ \mu_{Q}(b)\right) \\
& \text { as } P, Q \text { are } m \text {-polar PFIs of } A] \\
& =\left(p_{l} \circ \mu_{P}(a \diamond b) \wedge p_{l} \circ \mu_{Q}(a \diamond b)\right) \wedge\left(p_{l} \circ \mu_{P}(b) \wedge p_{l} \circ \mu_{Q}(b)\right) \\
& =p_{l} \circ \mu_{R}(a \diamond b) \wedge p_{l} \circ \mu_{R}(b), \\
& \quad p_{l} \circ \eta_{R}(a) \\
& =p_{l} \circ \eta_{P}(a) \wedge p_{l} \circ \eta_{Q}(a) \\
& \geqslant\left(p_{l} \circ \eta_{P}(a \diamond b) \wedge p_{l} \circ \eta_{P}(b)\right) \wedge\left(p_{l} \circ \eta_{Q}(a \diamond b) \wedge p_{l} \circ \eta_{Q}(b)\right) \\
& {[\text { as } P, Q \text { are } m \text {-polar PFIs of } A]} \\
& =\left(p_{l} \circ \eta_{P}(a \diamond b) \wedge p_{l} \circ \eta_{Q}(a \diamond b)\right) \wedge\left(p_{l} \circ \eta_{P}(b) \wedge p_{l} \circ \eta_{Q}(b)\right) \\
& =p_{l} \circ \eta_{R}(a \diamond b) \wedge p_{l} \circ \eta_{R}(b) \\
& \text { and } p_{l} \circ v_{R}(a) \\
& =p_{l} \circ v_{P}(a) \vee p_{l} \circ v_{Q}(a) \\
& \leqslant\left(p_{l} \circ v_{P}(a \diamond b) \vee p_{l} \circ v_{P}(b)\right) \vee\left(p_{l} \circ v_{Q}(a \diamond b) \vee p_{l} \circ v_{Q}(b)\right) \\
& {[\text { as } P, Q \text { are } m \text {-polar PFIs of } A]} \\
& =\left(p_{l} \circ v_{P}(a \diamond b) \vee p_{l} \circ v_{Q}(a \diamond b)\right) \vee\left(p_{l} \circ v_{P}(b) \vee p_{l} \circ v_{Q}(b)\right) \\
& =p_{l} \circ v_{R}(a \diamond b) \vee p_{l} \circ v_{R}(b), \forall a, b \in A \text { and } l=1,2, \ldots, m .
\end{aligned}
$$

Thus, $p_{l} \circ \mu_{R}(a) \geqslant p_{l} \circ \mu_{R}(a \diamond b) \wedge p_{l} \circ \mu_{R}(b), p_{l} \circ \eta_{R}(a) \geqslant p_{l} \circ$ $\eta_{R}(a \diamond b) \wedge p_{l} \circ \eta_{R}(b)$ and $p_{l} \circ v_{R}(a \diamond b) \vee p_{l} \circ v_{R}(b), \forall a, b \in A$ and for $l=1,2, \ldots, m$. Consequently, $R=P \cap Q$ is an $m$-polar PFI of $A$.

Proposition 8. Let $P=\left(\mu_{P}, \eta_{P}, v_{P}\right)$ and $Q=\left(\mu_{Q}, \eta_{Q}, v_{Q}\right)$ be two $m$-polar PFIs of a BCK algebra $(A, \diamond, 0)$. Then $P \times Q$ is an $m$-polar PFI of $A \times A$.

Proof. Proof is same as Proposition 7. So, it is omitted.

Proposition 9. Let $(A, \diamond, 0)$ be a $B C K$ algebra and $P=\left(\mu_{P}, \eta_{P}, v_{P}\right)$ be an m-polar PFI of $A$. Then $C_{\theta, \phi, \psi}(P)$ is a crisp ideal of $A$, provided that $P_{l} \circ \mu_{P}(0) \geqslant p_{l} \circ \theta, p_{l} \circ \eta_{P}(0) \geqslant p_{l} \circ \phi$ and $p_{l} \circ v_{P}(0) \leqslant p_{l} \circ \psi$ for $l=1,2, \ldots, m$.
Proof. Clearly, $C_{\theta, \phi, \psi}(P)$ contains at least one element. Let $a \diamond b$, $b \in C_{\theta, \phi, \psi}(P)$. Then $p_{l} \circ \mu_{P}(a \diamond b) \geqslant p_{l} \circ \theta, p_{l} \circ \eta_{P}(a \diamond b) \geqslant p_{l} \circ \phi$, $p_{l} \circ v_{P}(a \diamond b) \leqslant \psi$ and $p_{l} \circ \mu_{P}(b) \geqslant p_{l} \circ \theta, p_{l} \circ \eta_{P}(b) \geqslant p_{l} \circ \phi$, $p_{l} \circ v_{P}(b) \leqslant p_{l} \circ \psi$ for $l=1,2, \ldots, m$.

$$
\begin{aligned}
& \text { Now, } p_{l} \circ \mu_{P}(a) \\
& \geqslant p_{l} \circ \mu_{P}(a \diamond b) \wedge p_{l} \circ \mu_{P}(b) \\
& {[\text { because } P \text { is an } m \text {-polar PFI of } A]} \\
& \geqslant p_{l} \circ \theta \wedge p_{l} \circ \theta=p_{l} \circ \theta, \\
& p_{l} \circ \eta_{P}(a) \\
& \geqslant p_{l} \circ \eta_{P}(a \diamond b) \wedge p_{l} \circ \eta_{P}(b) \\
& {[\text { because } P \text { is an } m \text {-polar PFI of } A]} \\
& \geqslant p_{l} \circ \phi \wedge p_{l} \circ \phi=p_{l} \circ \phi \\
& \text { and } p_{l} \circ v_{P}(a) \\
& \leqslant p_{l} \circ v_{P}(a \diamond b) \vee p_{l} \circ v_{P}(b) \\
& {[\text { because } P \text { is an } m \text {-polar PFI of } A]} \\
& \leqslant p_{l} \circ \psi \vee p_{l} \circ \psi=p_{l} \circ \psi \text { for } l=1,2, \ldots . m
\end{aligned}
$$

Thus, $a \diamond b, b \in C_{\theta, \phi, \psi}(P) \Rightarrow a \in C_{\theta, \phi, \psi}(P)$. So, $C_{\theta, \phi, \psi}(P)$ is a crisp ideal of $A$.

Proposition 10. Let $(A, \diamond, 0)$ be a BCK algebra and $P=\left(\mu_{P}, \eta_{P}, v_{P}\right)$ be an $m$-polar PFS in $A$. Then $P$ is an m-polar PFI of $A$ if all $(\theta, \phi, \psi)$ cuts of $P$ are crisp ideals of $A$.

Proof. Let $a, b \in A$. Let $p_{l} \circ \mu_{P}(a \diamond b) \wedge p_{l} \circ \mu_{P}(b)=p_{l} \circ \theta, p_{l} \circ$ $\eta_{P}(a \diamond b) \wedge p_{l} \circ \eta_{P}(b)=p_{l} \circ \phi$ and $p_{l} \circ v_{P}(a \diamond b) \vee p_{l} \circ v_{P}(b)=p_{l} \circ \psi$ for $l=1,2, \ldots, m$. Clearly, $p_{l} \circ \theta \in[0,1], p_{l} \circ \phi \in[0,1]$ and $p_{l} \circ \psi \in[0,1]$ with $0 \leqslant p_{l} \circ \theta+p_{l} \circ \phi+p_{l} \circ \psi \leqslant 1$ for $l=1,2, \ldots, m$.

$$
\begin{aligned}
& \text { Now, } p_{l} \circ \mu_{P}(a \diamond b) \geqslant p_{l} \circ \mu_{P}(a \diamond b) \wedge p_{l} \circ \mu_{P}(b)=p_{l} \circ \theta \text {, } \\
& p_{l} \circ \eta_{P}(a \diamond b) \geqslant p_{l} \circ \mu_{P}(a \diamond b) \wedge p_{l} \circ \mu_{P}(b)=p_{l} \circ \phi \\
& \text { and } p_{l} \circ v_{P}(a \diamond b) \leqslant p_{l} \circ v_{P}(a \diamond b) \vee p_{l} \circ v_{P}(b)=p_{l} \circ \psi \\
& \text { for } l=1,2, \ldots, m \text {. } \\
& \text { Also, } p_{l} \circ \mu_{P}(b) \geqslant p_{l} \circ \mu_{P}(a \diamond b) \wedge p_{l} \circ \mu_{P}(b)=p_{l} \circ \theta \text {, } \\
& p_{l} \circ \eta_{P}(b) \geqslant p_{l} \circ \mu_{P}(a \diamond b) \wedge p_{l} \circ \mu_{P}(b)=p_{l} \circ \phi \\
& \text { and } p_{l} \circ v_{P}(b) \leqslant p_{l} \circ v_{P}(a \diamond b) \vee p_{l} \circ v_{P}(b)=p_{l} \circ \psi \\
& \text { for } l=1,2, \ldots, m \text {. }
\end{aligned}
$$

Thus, $a \diamond b$ and $b \in C_{\theta, \phi, \psi}(P)$. Since $C_{\theta, \phi, \psi}(P)$ is a crisp ideal of $A$ therefore $a \diamond b \in C_{\theta, \phi, \psi}(P)$ and $b \in C_{\theta, \phi, \psi}(P) \Rightarrow a \in C_{\theta, \phi, \psi}(P)$.

Therefore, $p_{l} \circ \mu_{P}(a) \geqslant p_{l} \circ \theta=p_{l} \circ \mu_{P}(a \diamond b) \wedge p_{l} \circ \mu_{P}(b), p_{l} \circ \eta_{P}(a) \geqslant$ $p_{l} \circ \phi=p_{l} \circ \eta_{P}(a \diamond b) \wedge p_{l} \circ \eta_{P}(b)$ and $p_{l} \circ v_{P}(a) \leqslant p_{l} \circ \psi=p_{l} \circ$ $v_{P}(a \diamond b) \vee p_{l} \circ v_{P}(b)$ for $l=1,2, . ., m$.

Since $a, b$ are arbitrary elements of $A$ therefore $p_{l} \circ \mu_{P}(a) \geqslant p_{l} \circ$ $\mu_{P}(a \diamond b) \wedge p_{l} \circ \mu_{P}(b), p_{l} \circ \eta_{P}(a) \geqslant p_{l} \circ \eta_{P}(a \diamond b) \wedge p_{l} \circ \eta_{P}(b)$ and $p_{l} \circ v_{P}(a) \leqslant p_{l} \circ v_{P}(a \diamond b) \vee p_{l} \circ v_{P}(b)$ for all $a, b \in A$ and for $l=$ $1,2, \ldots, m$. Hence, $P$ is an $m$-polar PFI of $A$.

\section{PRE-IMAGE AND IMAGE PFI UNDER HOMOMORPHISM OF BCK ALGEBRA}

In the current section, we explore some properties of $m$-polar PFI of BCK algebra under homomorphism of BCK algebra.

Definition 17. Let $\left(A_{1}, \diamond, 0\right)$ and $\left(A_{2}, *, 0\right)$ be two BCK algebras. Then a mapping $h: A_{1} \rightarrow A_{2}$ is said to be homomorphism if $h(a \diamond b)=h(a) * h(b)$ for all $a, b \in A_{1}$. 
It is observed that $h(a) * h(a)=0$ i.e. $h(a \diamond a)=0$ i.e. $h(0)=0$.

Proposition 11. Let $\left(A_{1}, \diamond, 0\right)$ and $\left(A_{2}, *, 0\right)$ be two BCK algebras and $Q=\left(\mu_{Q}, \eta_{Q}, v_{Q}\right)$ be an m-polar PFI of $A_{2}$. Then for a $B C K$ algebra homomorphism $h: A_{1} \rightarrow A_{2}, h^{-1}(Q)$ is an m-polar PFI of $A_{1}$.

Proof. Let $h^{-1}(Q)=\left(\mu_{h^{-1}(Q)}, \eta_{h^{-1}(Q)}, v_{h^{-1}(Q)}\right)$, where $\mu_{h^{-1}(Q)}=$ $\mu_{Q}(h(a)), \eta_{h^{-1}(Q)}(a)=\eta_{Q}(h(a))$ and $v_{h^{-1}(Q)}(a)=v_{Q}(h(a))$ for all $a \in A_{1}$.

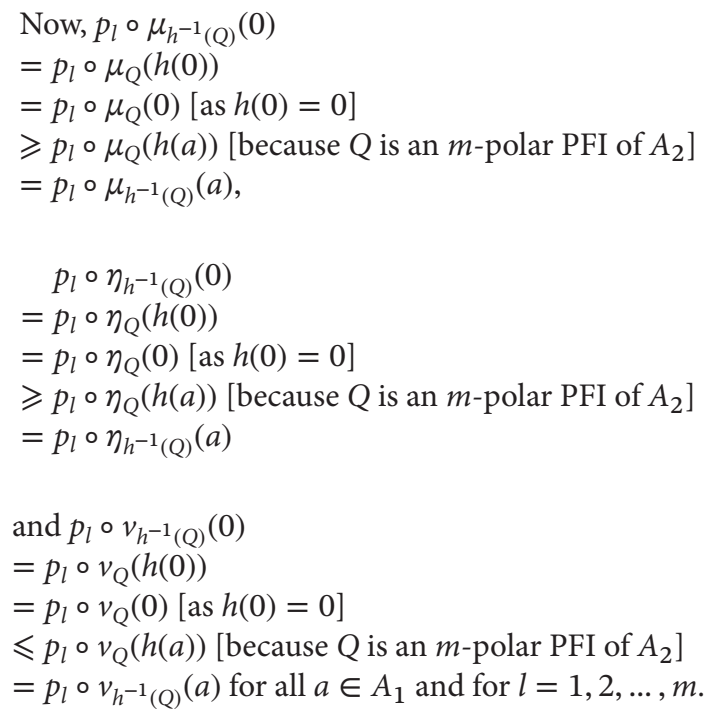

Thus, $p_{l} \circ \mu_{h^{-1}(Q)}(0) \geqslant p_{l} \circ \mu_{h^{-1}(Q)}(a), p_{l} \circ \eta_{h^{-1}(Q)}(0) \geqslant p_{l} \circ \eta_{h^{-1}(Q)}(a)$ and $p_{l} \circ v_{h^{-1}(Q)}(0) \leqslant p_{l} \circ v_{h^{-1}(Q)}(a)$ for all $a \in A_{1}$ and for $l=$ $1,2, \ldots, m$.

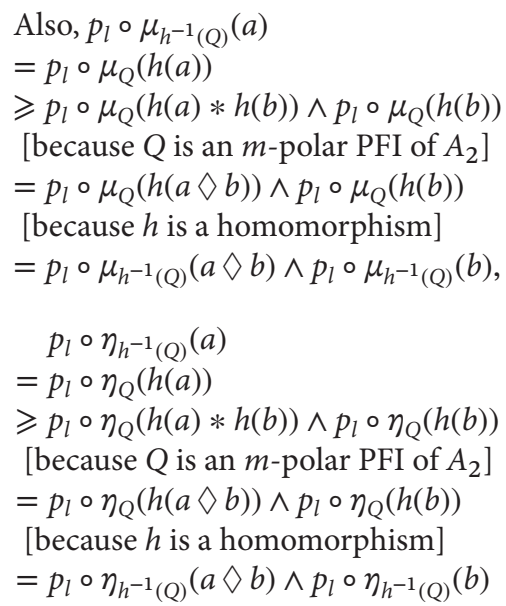

and $p_{l} \circ v_{h^{-1}(Q)}(a)$

$=p_{l} \circ v_{Q}(h(a))$

$\leqslant p_{l} \circ v_{Q}(h(a) * h(b)) \vee p_{l} \circ v_{Q}(h(b))$

[because $Q$ is an $m$-polar PFI of $A_{2}$ ]

$=p_{l} \circ v_{Q}(h(a \diamond b)) \vee p_{l} \circ v_{Q}(h(b))$

[because $h$ is a homomorphism]

$=p_{l} \circ v_{h^{-1}(Q)}(a \diamond b) \vee p_{l} \circ v_{h^{-1}(Q)}(b)$ for all $a, b \in A_{1}$ and for $l=1,2, \ldots, m$.

Thus, $p_{l} \circ \mu_{h^{-1}(Q)}(a) \geqslant p_{l} \circ \mu_{h^{-1}(Q)}(a \diamond b) \wedge p_{l} \circ \mu_{h^{-1}(Q)}(b), p_{l} \circ$ $\eta_{h^{-1}(Q)}(a) \geqslant p_{l} \circ \eta_{h^{-1}}(a \diamond b) \wedge p_{l} \circ \eta_{h^{-1}(Q)}(b)$ and $p_{l} \circ v_{h^{-1}(Q)}(a) \leqslant p_{l} \circ$ $v_{h^{-1}(\mathrm{Q})}(a \diamond b) \vee p_{l} \circ v_{h^{-1}(\mathrm{Q})}(b)$ for all $a, b \in A_{1}$ and for $l=1,2, \ldots, m$. Hence, $h^{-1}(Q)$ is an $m$-polar PFI of $A_{1}$.
Proposition 12. Let $\left(A_{1}, \diamond\right)$ and $\left(A_{2}, *\right)$ be two BCK algebras and $P=\left(\mu_{P}, \eta_{P}, v_{P}\right)$ be an m-polar PFI of $A_{1}$. Then for a bijective homomorphism $h: A_{1} \rightarrow A_{2}, h(P)$ is an m-polar PFI of $A_{2}$.

Proof. Let $h(P)=\left(\mu_{h(P)}, \eta_{h(P)}, v_{h(P)}\right)$. Now, let $b \in A_{2}$.

$$
\begin{aligned}
\text { Then } p_{l} \circ \mu_{h(P)}(b) & =\underset{a \in h^{-1}(b)}{\vee} p_{l} \circ \mu_{P}(a), \\
p_{l} \circ \eta_{h(P)}(b) & =\underset{a \in h^{-1}(b)}{\wedge} p_{l} \circ \eta_{P}(a) \\
\text { and } p_{l} \circ v_{h(P)}(b) & =\underset{a \in h^{-1}(b)}{\wedge} p_{l} \circ v_{P}(a) \text { for } l=1,2, . ., m .
\end{aligned}
$$

Since $h$ is bijective therefore $h^{-1}(b)$ must be a singleton set. So, for $b \in A_{2}$, there exists an unique $a \in A_{1}$ such that $a=h^{-1}(b)$ i.e. $h(a)=b$. Thus, in this case, $p_{l} \circ \mu_{h(P)}(b)=p_{l} \circ \mu_{h(P)}(h(a))=p_{l} \circ \mu_{P}(a)$, $p_{l} \circ \eta_{h(P)}(b)=p_{l} \circ \eta_{h(P)}(h(a))=p_{l} \circ \eta_{P}(a)$ and $p_{l} \circ v_{h(P)}(b)=p_{l} \circ$ $v_{h(P)}(h(a))=p_{l} \circ v_{P}(a)$ for $l=1,2, \ldots, m$.

$$
\begin{aligned}
& \text { Now, } p_{l} \circ \mu_{h(P)}(0) \\
&=p_{l} \circ \mu_{h(P)}(h(0))[\text { as } h(0)=0] \\
&=p_{l} \circ \mu_{P}(0) \\
& \geqslant p_{l} \circ \mu_{P}(a) \\
&= p_{l} \circ \mu_{h(P)}(h(a)) \\
&= p_{l} \circ \mu_{h(P)}(b), \\
& p_{l} \circ \eta_{h(P)}(0) \\
&= p_{l} \circ \eta_{h(P)}(h(0))[\text { as } h(0)=0] \\
&= p_{l} \circ \eta_{P}(0) \\
& \geqslant p_{l} \circ \eta_{P}(a) \\
&= p_{l} \circ \eta_{h(P)}(h(a)) \\
&= p_{l} \circ \eta_{h(P)}(b) \\
& \text { and } p_{l} \circ v_{h(P)}(0) \\
&=p_{l} \circ v_{h(P)}(h(0))[\text { as } h(0)=0] \\
&=p_{l} \circ v_{P}(0) \\
& \leqslant p_{l} \circ v_{P}(a) \\
&=p_{l} \circ v_{h(P)}(h(a)) \\
&=p_{l} \circ v_{h(P)}(b) \text { for } l=1,2, \ldots, m .
\end{aligned}
$$

Since $b$ is an arbitrary element of $A_{2}$ therefore $p_{l} \circ \mu_{h(P)}(0) \geqslant p_{l} \circ$ $\mu_{h(P)}(b), p_{l} \circ \eta_{h(P)}(0) \geqslant p_{l} \circ \eta_{h(P)}(b)$ and $p_{l} \circ v_{h(P)}(0) \leqslant p_{l} \circ v_{h(P)}(b)$ for all $b \in A_{2}$ and for $l=1,2, \ldots, m$.

$$
\begin{aligned}
& \text { Also, } p_{l} \circ \mu_{h(P)}(b) \\
& =p_{l} \circ \mu_{h(P)}(h(a)) \text { [where } b=h(a) \text { for unique } a \in A_{1} \text { ] } \\
& =p_{l} \circ \mu_{P}(a) \\
& \geqslant p_{l} \circ \mu_{P}(a \diamond c) \wedge p_{l} \circ \mu_{P}(c) \\
& {\left[\text { as } P \text { is an } m \text {-polar PFI of } A_{1}\right]} \\
& =p_{l} \circ \mu_{h(P)}(h(a \diamond c)) \wedge p_{l} \circ \mu_{h(P)}(h(c)) \\
& =p_{l} \circ \mu_{h(P)}(h(a) * h(c)) \wedge p_{l} \circ \mu_{h(P)}(h(c)) \\
& {[\text { as } h \text { is a homomorphism] }} \\
& =p_{l} \circ \mu_{h(P)}(b * h(c)) \wedge p_{l} \circ \mu_{h(P)}(h(c)), \\
& \\
& \quad p_{l} \circ \eta_{h(P)}(b) \\
& =p_{l} \circ \eta_{h(P)}(h(a))\left[\text { where } b=h(a) \text { for unique } a \in A_{1}\right] \\
& =p_{l} \circ \eta_{P}(a) \\
& \geqslant p_{l} \circ \eta_{P}(a \diamond c) \wedge p_{l} \circ \eta_{P}(c) \\
& {\left[\text { as } P \text { is an } m \text {-polar PFI of } A_{1}\right]} \\
& =p_{l} \circ \eta_{h(P)}(h(a \diamond c)) \wedge p_{l} \circ \eta_{h(P)}(h(c)) \\
& =p_{l} \circ \eta_{h(P)}(h(a) * h(c)) \wedge p_{l} \circ \eta_{h(P)}(h(c)) \\
& {[\text { as } h \text { is a homomorphism] }} \\
& =p_{l} \circ \eta_{h(P)}(b * h(c)) \wedge p_{l} \circ \eta_{h(P)}(h(c))
\end{aligned}
$$


and $p_{l} \circ v_{h(P)}(b)$

$=p_{l} \circ v_{h(P)}(h(a))\left[\right.$ where $b=h(a)$ for unique $\left.a \in A_{1}\right]$

$=p_{l} \circ v_{P}(a)$

$\leqslant p_{l} \circ v_{P}(a \diamond c) \vee p_{l} \circ v_{P}(c)$

[as $P$ is an $m$-polar PFI of $A_{1}$ ]

$=p_{l} \circ v_{h(P)}(h(a \diamond c)) \vee p_{l} \circ v_{h(P)}(h(c))$

$=p_{l} \circ v_{h(P)}(h(a) * h(c)) \vee p_{l} \circ v_{h(P)}(h(c))$

[as $h$ is a homomorphism]

$=p_{l} \circ v_{h(P)}(b * h(c)) \vee p_{l} \circ v_{h(P)}(h(c))$ for all $c \in A_{1}$ and for $l=1,2, \ldots, m$.

Thus, $p_{l} \circ \mu_{h(P)}(b) \geqslant p_{l} \circ \mu_{h(P)}(b * h(c)) \wedge p_{l} \circ \mu_{h(P)}(h(c)), p_{l} \circ \eta_{h(P)}(b) \geqslant$ $p_{l} \circ \eta_{h(P)}(b * h(c)) \wedge p_{l} \circ \eta_{h(P)}(h(c))$ and $p_{l} \circ v_{h(P)}(b) \leqslant p_{l} \circ v_{h(P)}(b * h(c)) \vee$ $p_{l} \circ v_{h(P)}(h(c))$ for all $c \in A_{1}$ and for $l=1,2, \ldots, m$. Since $h$ is bijective therefore $h\left(A_{1}\right)=A_{2}$. So, for all $c \in A_{1}, h(c)$ can capture all the elements of $A_{2}$. Letting $d=h(c)$, it is observed that the inequalities hold for all $d \in A_{2}$. Since $b$ is arbitrary therefore we obtain that $p_{l} \circ \mu_{h(P)}(b) \geqslant p_{l} \circ \mu_{h(P)}(b \diamond d) \wedge p_{l} \circ \mu_{h(P)}(d), p_{l} \circ \eta_{h(P)}(b) \geqslant p_{l} \circ$ $\eta_{h(P)}(b \diamond d) \wedge p_{l} \circ \eta_{h(P)}(d)$ and $p_{l} \circ v_{h(P)}(b) \leqslant p_{l} \circ v_{h(P)}(b \diamond d) \vee p_{l} \circ v_{h(P)}(d)$ for all $b, d \in A_{2}$ and for $l=1,2, \ldots, m$. Hence, $h(P)$ is an $m$-polar PFI of $A_{2}$.

\section{6. $m$-POLAR PFII}

The current section introduces the concept of implicative BCK algebra, $m$-polar PFII of a BCK algebra and studies some properties related to these. We also investigate a relationship between $m$-polar PFI and $m$-polar PFII of a BCK algebra.

Definition 18. A BCK algebra $(A, \diamond, 0)$ is said to be implicative if $a=(a \diamond b) \diamond a$ for all $a, b \in A$.

Proposition 13. An $m$-polar PFS $P=\left(\mu_{P}, \eta_{P}, v_{P}\right)$ in a $B C K$ algebra $(A, \diamond, 0)$ is said to be m-polar PFII of $A$ if the below stated conditions are meet.

i. $\quad p_{l} \circ \mu_{P}(0) \geqslant p_{l} \circ \mu_{P}(a), p_{l} \circ \eta_{P}(0) \geqslant p_{l} \circ \eta_{P}(a)$ and $p_{l} \circ v_{P}(0) \leqslant$ $p_{l} \circ v_{P}(a)$

ii. $p_{l} \circ \mu_{P}(a) \geqslant p_{l} \circ \mu_{P}\{(a \diamond(b \diamond a)) \diamond c\} \wedge p_{l} \circ \mu_{P}(c), p_{l} \circ \eta_{P}(a) \geqslant$ $p_{l} \circ \eta_{P}\{(a \diamond(b \diamond a)) \diamond c\} \wedge p_{l} \circ \eta_{P}(c)$ and $p_{l} \circ v_{P}(a) \leqslant p_{l} \circ$ $v_{P}\{(a \diamond(b \diamond a)) \diamond c\} \vee p_{l} \circ v_{P}(c)$ for all $a, b, c \in A$ and for $l=1,2, \ldots, m$

Example 3. Let us consider the BCK algebra $(A, \diamond)$ as follows:

\begin{tabular}{llllll}
\hline$\diamond$ & 0 & $p$ & $q$ & $r$ & $s$ \\
0 & 0 & 0 & 0 & 0 & 0 \\
$p$ & $p$ & 0 & $p$ & 0 & 0 \\
$q$ & $q$ & $q$ & 0 & 0 & 0 \\
$r$ & $r$ & $r$ & $r$ & 0 & 0 \\
$s$ & $s$ & $r$ & $s$ & $p$ & 0 \\
\hline
\end{tabular}

Let us consider a 3-polar PFS $P=\left(\mu_{P}, \eta_{P}, v_{P}\right)$ as follows:

$$
\mu_{P}(a)= \begin{cases}(0.39,0.41,0.42), & \text { if } a=0, p, q \\ (0.25,0.27,0.3), & \text { if } a=r, s\end{cases}
$$

$$
\eta_{P}(a)= \begin{cases}(0.37,0.39,0.4), & \text { if } a=0, p, q \\ (0.29,0.33,0.35), & \text { if } a=r, s\end{cases}
$$

and

$$
v_{P}(a)= \begin{cases}(0.14,0.17,0.18), & \text { if } a=0, p, q \\ (0.3,0.32,0.35), & \text { if } a=r, s\end{cases}
$$

It can be easily shown that $P$ is a 3-polar PFII of $A$.

Proposition 14. Every m-polar PFII of a BCK algebra $(A, \diamond, 0)$ is an $m$-polar PFI of $A$.

Proof. Let $P=\left(\mu_{P}, \eta_{P}, v_{P}\right)$ be an $m$-polar PFII of $A$.

Then $p_{l} \circ \mu_{P}(a) \geqslant p_{l} \circ \mu_{P}\{(a \diamond(b \diamond a)) \diamond c\} \wedge p_{l} \circ \mu_{P}(c)$,

$p_{l} \circ \eta_{P}(a) \geqslant p_{l} \circ \eta_{P}\{(a \diamond(b \diamond a)) \diamond c\} \wedge p_{l} \circ \eta_{P}(c)$

and $p_{l} \circ v_{P}(a) \leqslant p_{l} \circ v_{P}\{(a \diamond(b \diamond a)) \diamond c\} \vee p_{l} \circ v_{P}(c)$

for all $a, b, c \in A$ and for $l=1,2, \ldots, m$.

Setting $b=a$, it is obtained that

$$
\begin{aligned}
p_{l} \circ \mu_{P}(a) & \geqslant p_{l} \circ \mu_{P}\{(a \diamond(a \diamond a)) \diamond c\} \wedge p_{l} \circ \mu_{P}(c) \\
& =p_{l} \circ \mu_{P}\{(a \diamond 0) \diamond c\} \wedge p_{l} \circ \mu_{P}(c) \\
& =p_{l} \circ \mu_{P}(a \diamond c) \wedge p_{l} \circ \mu_{P}(c)
\end{aligned}
$$

[by Proposition 1]

$$
\begin{aligned}
p_{l} \circ \eta_{P}(a) & \geqslant p_{l} \circ \eta_{P}\{(a \diamond(a \diamond a)) \diamond c\} \wedge p_{l} \circ \eta_{P}(c) \\
& =p_{l} \circ \eta_{P}\{(a \diamond 0) \diamond c\} \wedge p_{l} \circ \eta_{P}(c) \\
& =p_{l} \circ \eta_{P}(a \diamond c) \wedge p_{l} \circ \eta_{P}(c)
\end{aligned}
$$

[by Proposition 1 ]

$$
\text { and } \begin{aligned}
p_{l} \circ v_{P}(a) \leqslant & p_{l} \circ v_{P}\{(a \diamond(a \diamond a)) \diamond c\} \vee p_{l} \circ v_{P}(c) \\
= & p_{l} \circ v_{P}\{(a \diamond 0) \diamond c\} \vee p_{l} \circ v_{P}(c) \\
= & p_{l} \circ v_{P}(a \diamond c) \vee p_{l} \circ v_{P}(c) \\
& \quad[\text { by Proposition } 1] \\
& \quad \text { for all } a, c \in A \text { and for } l=1,2, \ldots, m .
\end{aligned}
$$

Therefore, $P$ is an $m$-polar PFI of $A$.

The above proposition does not hold in reverse direction i.e. an $m$-polar PFI of a BCK algebra is not necessarily $m$-polar PFII which is clear from the following example. It is necessary to mention that in an implicative BCK algebra, the converse of the above proposition holds which is shown through Proposition 15.

Example 4. Now, let us consider a 3-polar PFS $P=\left(\mu_{P}, \eta_{P}, v_{P}\right)$ in BCK algebra $A$ given in Example 3 as follows:

$$
\begin{aligned}
& \mu_{P}(a)= \begin{cases}(0.42,0.43,0.45), & \text { if } a=0, q \\
(0.25,0.27,0.3), & \text { if } a=p, r, s\end{cases} \\
& \eta_{P}(a)= \begin{cases}(0.3,0.33,0.35), & \text { if } a=0, q \\
(0.15,0.18,0.2), & \text { if } a=p, r, s\end{cases}
\end{aligned}
$$

and

$$
v_{P}(a)= \begin{cases}(0.14,0.16,0.2), & \text { if } a=0, q \\ (0.45,0.48,0.5), & \text { if } a=p, r, s\end{cases}
$$


It is clear that $(0.25,0.27,0.3)=\mu_{P}(p) \nsupseteq \mu_{P}\{(p \diamond(r \diamond p)) \diamond q\} \wedge$ $\mu_{P}(q)=(0.42,0.43,0.45) \wedge(0.42,0.43,0.45)=(0.42,0.43,0.45)$, $(0.15,0.18,0.2)=\eta_{P}(p) \nsupseteq \eta_{P}\{(p \diamond(r \diamond p)) \diamond q\} \wedge \eta_{P}(q)=$ $(0.3,0.33,0.35) \wedge(0.3,0.33,0.35)=(0.3,0.33,0.35)$ and $(0.45,0.48,0.5)=v_{P}(p) \not \leq v_{P}\{(p \diamond(q \diamond p)) \diamond q\} \wedge v_{P}(q)=$ $(0.14,0.16,0.2) \vee(0.14,0.16,0.2)=(0.14,0.16,0.2)$. Thus, $P$ is not 3-polar PFII although it is a 3-polar PFI of $A$.

Proposition 15. In an implicative BCK algebra, every m-polar PFI is m-polar PFII.

Proof. Let $(A, \diamond, 0)$ be an implicative BCK algebra. Therefore, $a=$ $(a \diamond b) \diamond a$ for all $a, b \in A$. Let $P=\left(\mu_{P}, \eta_{P}, v_{P}\right)$ be an $m$-polar PFI of $A$. Then

$$
\begin{aligned}
p_{l} \circ \mu_{P}(a) \geqslant & p_{l} \circ \mu_{P}(a \diamond c) \wedge p_{l} \circ \mu_{P}(c) \\
= & p_{l} \circ \mu_{P}\{((a \diamond b) \diamond a) \diamond c\} \wedge p_{l} \circ \mu_{P}(c), \\
p_{l} \circ \eta_{P}(a) \geqslant & p_{l} \circ \eta_{P}(a \diamond c) \wedge p_{l} \circ \eta_{P}(c) \\
= & p_{l} \circ \eta_{P}\{((a \diamond b) \diamond a) \diamond c\} \wedge p_{l} \circ \eta_{P}(c) \\
\text { and } p_{l} \circ v_{P}(a) \leqslant & p_{l} \circ v_{P}(a \diamond c) \vee p_{l} \circ v_{P}(c) \\
= & p_{l} \circ v_{P}\{((a \diamond b) \diamond a) \diamond c\} \vee p_{l} \circ v_{P}(c) \\
& \quad \text { for all } a, b, c \in A \text { and for } l=1,2, \ldots, m .
\end{aligned}
$$

Thus, $P$ is an $m$-polar PFII of $A$.

Proposition 16. Let $(A, \diamond, 0)$ be a $B C K$ algebra and $P=\left(\mu_{P}, \eta_{P}, v_{P}\right)$ be an m-polar PFII of $A$. Then $C_{\theta, \phi, \psi}(P)$ is an implicative ideal of $A$, provided that $p_{l} \circ \mu_{P}(0) \geqslant p_{l} \circ \theta, p_{l} \circ \eta_{P}(0) \geqslant p_{l} \circ \phi$ and $p_{l} \circ v_{P}(0) \leqslant p_{l} \circ \psi$ for $l=1,2, \ldots . m$.

Proof. Clearly, $C_{\theta, \phi, \psi}(P)$ contains at least one element. Let $((a \diamond b) \diamond a) \diamond c, c \in C_{\theta, \phi, \psi}(P)$. Then $p_{l} \circ \mu_{P}\{((a \diamond b) \diamond a) \diamond c\} \geqslant$ $p_{l} \circ \theta, p_{l} \circ \eta_{P}\{((a \diamond b) \diamond a) \diamond c\} \geqslant p_{l} \circ \phi, p_{l} \circ v_{P}\{((a \diamond b) \diamond a) \diamond c\} \leqslant p_{l} \circ \psi$ and $p_{l} \circ \mu_{P}(c) \geqslant p_{l} \circ \theta, p_{l} \circ \eta_{P}(c) \geqslant p_{l} \circ \phi, p_{l} \circ v_{P}(c) \leqslant p_{l} \circ \psi$ for $l=1,2, \ldots, m$.

$$
\begin{aligned}
& \text { Now, } p_{l} \circ \mu_{P}(a) \geqslant p_{l} \circ \mu_{P}\{((a \diamond b) \diamond a) \diamond c\} \wedge p_{l} \circ \mu_{P}(c) \\
& \text { [because } P \text { is an } m \text {-polar PFII of } A \text { ] } \\
& \geqslant p_{l} \circ \theta \wedge p_{l} \circ \theta=p_{l} \circ \theta \text {, } \\
& p_{l} \circ \eta_{P}(a) \geqslant p_{l} \circ \eta_{P}\{((a \diamond b) \diamond a) \diamond c\} \wedge p_{l} \circ \eta_{P}(c) \\
& \text { [because } P \text { is an } m \text {-polar PFII of } A \text { ] } \\
& \geqslant p_{l} \circ \phi \wedge p_{l} \circ \phi=p_{l} \circ \phi \\
& \text { and } p_{l} \circ v_{P}(a) \leqslant p_{l} \circ v_{P}\{((a \diamond b) \diamond a) \diamond c\} \vee p_{l} \circ v_{P}(c) \\
& \text { [because } P \text { is an } m \text {-polar PFII of } A \text { ] } \\
& \leqslant p_{l} \circ \psi \vee p_{l} \circ \psi=p_{l} \circ \psi \\
& \text { for } l=1,2, \ldots, m \text {. }
\end{aligned}
$$

Thus, it is observed that $\{((a \diamond b) \diamond a) \diamond c\}, c \in C_{\theta, \phi, \psi}(P) \Rightarrow a \in$ $C_{\theta, \phi, \psi}(P)$. So, $C_{\theta, \phi, \psi}(P)$ is an implicative ideal of $A$.

Proposition 17. Let $(A, \diamond, 0)$ be a $B C K$ algebra and $P=\left(\mu_{P}, \eta_{P}, v_{P}\right)$ be an $m$-polar PFS in $A$. Then $P$ is an $m$-polar PFII of $A$ if all $(\theta, \phi, \psi)$ cuts of $P$ are implicative ideals of $A$.

Proof. Let $a, b \in A$. Let $p_{l} \circ \mu_{P}\{((a \diamond b) \diamond a) \diamond c\} \wedge p_{l} \circ \mu_{P}(c)=$ $p_{l} \circ \theta, p_{l} \circ \eta_{P}\{((a \diamond b) \diamond a) \diamond c\} \wedge p_{l} \circ \eta_{P}(c)=p_{l} \circ \phi$ and $p_{l} \circ$ $v_{P}\{((a \diamond b) \diamond a) \diamond c\} \vee p_{l} \circ v_{P}(c)=p_{l} \circ \psi$ for $l=1,2, \ldots, m$. Clearly, $p_{l} \circ \theta \in[0,1], p_{l} \circ \phi \in[0,1]$ and $p_{l} \circ \psi \in[0,1]$ with $0 \leqslant$ $p_{l} \circ \theta+p_{l} \circ \phi+p_{l} \circ \psi \leqslant 1$ for $l=1,2, \ldots, m$.

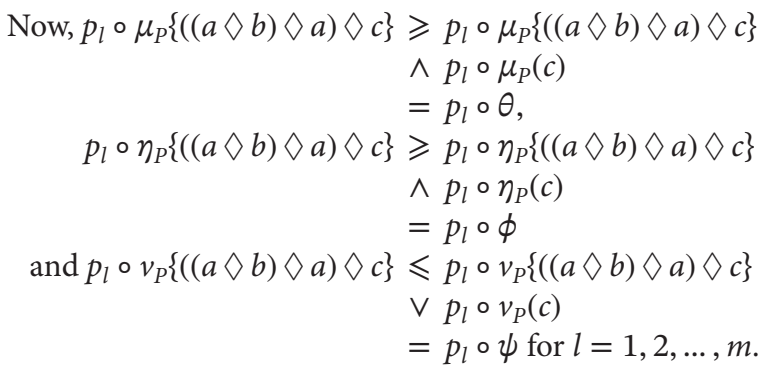

$$
\text { Also, } \begin{aligned}
p_{l} \circ \mu_{P}(c) & \geqslant p_{l} \circ \mu_{P}\{((a \diamond b) \diamond a) \diamond c\} \\
& \wedge p_{l} \circ \mu_{P}(c)=p_{l} \circ \theta \\
p_{l} \circ \eta_{P}(c) & \geqslant p_{l} \circ \eta_{P}\{((a \diamond b) \diamond a) \diamond c\} \\
& \wedge p_{l} \circ \eta_{P}(c)=p_{l} \circ \phi \\
\text { and } p_{l} \circ v_{P}(c) & \leqslant p_{l} \circ v_{P}\{((a \diamond b) \diamond a) \diamond c\} \\
& \vee p_{l} \circ v_{P}(c)=p_{l} \circ \psi \text { for } l=1,2, \ldots, m .
\end{aligned}
$$

Thus, $((a \diamond b) \diamond a) \diamond c$ and $c \in C_{\theta, \phi, \psi}(P)$. Since $C_{\theta, \phi, \psi}(P)$ is an implicative ideal of $A$ therefore $a \in C_{\theta, \phi, \psi}(P)$.

Therefore, $p_{l} \circ \mu_{P}(a) \geqslant p_{l} \circ \theta=p_{l} \circ \mu_{P}\{((a \diamond b) \diamond a) \diamond c\} \wedge p_{l} \circ \mu_{P}(c)$, $p_{l} \circ \eta_{P}(a) \geqslant p_{l} \circ \phi=p_{l} \circ \eta_{P}\{((a \diamond b) \diamond a) \diamond c\} \wedge p_{l} \circ \eta_{P}(c)$ and $p_{l} \circ v_{P}(a) \leqslant$ $p_{l} \circ \psi=p_{l} \circ v_{P}\{((a \diamond b) \diamond a) \diamond c\} \vee p_{l} \circ v_{P}(c)$ for $l=1,2, \ldots, m$.

Since $a, b, c$ are arbitrary elements of $A$ therefore $p_{l} \circ \mu_{P}(a) \geqslant p_{l} \circ$ $\mu_{P}\{((a \diamond b) \diamond a) \diamond c\} \wedge p_{l} \circ \mu_{P}(c), p_{l} \circ \eta_{P}(a) \geqslant p_{l} \circ \eta_{P}\{((a \diamond b) \diamond a) \diamond c\} \wedge$ $p_{l} \circ \eta_{P}(c)$ and $p_{l} \circ v_{P}(a) \leqslant p_{l} \circ v_{P}\{((a \diamond b) \diamond a) \diamond c\} \vee p_{l} \circ v_{P}(c)$ for all $a, b, c \in A$ and for $l=1,2, \ldots, m$. Hence, $P$ is an $m$-polar PFII of $A$.

Proposition 18. Let $S_{1}$ and $S_{2}$ be two ideals of a BCK algebra $(A, \diamond, 0)$ such that $S_{1} \subseteq S_{2}$. If $S_{1}$ is implicative then $S_{2}$ also.

Proposition 19. Let $P_{1}$ and $P_{2}$ be two m-polar PFIs of a BCK algebra $(A, \diamond, 0)$ with $P_{1} \subseteq P_{2}$. If $P_{1}$ is $m$-polar PFII of $A$ then $P_{2}$ also.

Proof. Let $a \in C_{\theta, \phi, \psi}\left(P_{1}\right)$. Then $p_{l} \circ \mu_{P_{1}}(a) \geqslant p_{l} \circ \theta, p_{l} \circ \eta_{P_{1}}(a) \geqslant$ $p_{l} \circ \phi$ and $p_{l} \circ v_{P_{1}}(a) \leqslant p_{l} \circ \psi$ for $l=1,2, \ldots, m$. Now, $P_{1} \subseteq P_{2} \Rightarrow$ $p_{l} \circ \mu_{P_{1}}(a) \leqslant p_{l} \circ \mu_{P_{2}}(a), p_{l} \circ \eta_{P_{1}}(a) \leqslant p_{l} \circ \eta_{P_{2}}(a)$ and $p_{l} \circ v_{P_{1}}(a) \geqslant$ $p_{l} \circ v_{P_{2}}(a)$ for $l=1,2, \ldots, m$. It follows that $p_{l} \circ \mu_{P_{2}}(a) \geqslant p_{l} \circ \theta$, $p_{l} \circ \eta_{P_{2}}(a) \geqslant p_{l} \circ \phi$ and $p_{l} \circ v_{P_{2}}(a) \leqslant p_{l} \circ \psi$ for $l=1,2, \ldots, m$. Thus, $a \in C_{\theta, \phi, \psi}\left(P_{2}\right)$. As a result, $C_{\theta, \phi, \psi}\left(P_{1}\right) \subseteq C_{\theta, \phi, \psi}\left(P_{2}\right)$. Since $P_{1}$ is an $m$-polar PFII of $A$ therefore $C_{\theta, \phi, \psi}\left(P_{1}\right)$ is implicative ideal of $A$ by Proposition 16. By Proposition 18, $C_{\theta, \phi, \psi}\left(P_{2}\right)$ is implicative ideal of $A$. Therefore, by Proposition $17, P_{2}$ is an $m$-polar PFII of $A$.

Proposition 20. Let $P=\left(\mu_{P}, \eta_{P}, v_{P}\right)$ be an m-polar PFI of a BCK algebra $A$. Then the below stated statements are equivalent.

i. $\quad P$ is $m$-polar PFII.

ii. $\quad p_{l} \circ \mu_{P}(a) \geqslant p_{l} \circ \mu_{P}(a \diamond(b \diamond a)), p_{l} \circ \eta_{P}(a) \geqslant p_{l} \circ \eta_{P}(a \diamond(b \diamond a))$ and $p_{l} \circ v_{P}(a) \leqslant p_{l} \circ v_{P}(a \diamond(b \diamond a))$ for all $a, b \in A$ and for $l=1,2, \ldots, m$.

iii. $\quad p_{l} \circ \mu_{P}(a)=p_{l} \circ \mu_{P}(a \diamond(b \diamond a)), p_{l} \circ \eta_{P}(a)=p_{l} \circ \eta_{P}(a \diamond(b \diamond a))$ and $p_{l} \circ v_{P}(a)=p_{l} \circ v_{P}(a \diamond(b \diamond a))$ for all $a, b \in A$ and for $l=1,2, \ldots, m$. 
Proof. $(i) \Rightarrow$ (ii): Since $P$ is an $m$-polar PFII of $A$, therefore,

$$
\begin{aligned}
p_{l} \circ \mu_{P}(a) \geqslant & p_{l} \circ \mu_{P}\{(a \diamond(b \diamond a)) \diamond 0\} \wedge p_{l} \circ \mu_{P}(0) \\
= & p_{l} \circ \mu_{P}(a \diamond(b \diamond a)) \wedge p_{l} \circ \mu_{P}(0) \\
& {[\text { by Proposition } 1] } \\
= & p_{l} \circ \mu_{P}(a \diamond(b \diamond a)) \\
p_{l} \circ \eta_{P}(a) \geqslant & p_{l} \circ \eta_{P}\{(a \diamond(b \diamond a)) \diamond 0\} \wedge p_{l} \circ \eta_{P}(0) \\
= & p_{l} \circ \eta_{P}(a \diamond(b \diamond a)) \wedge p_{l} \circ \eta_{P}(0) \\
& {[\text { by Proposition } 1] } \\
= & p_{l} \circ \eta_{P}(a \diamond(b \diamond a)) \\
\text { and } p_{l} \circ v_{P}(a) \leqslant & p_{l} \circ v_{P}\{(a \diamond(b \diamond a)) \diamond 0\} \vee p_{l} \circ v_{P}(0) \\
= & p_{l} \circ v_{P}(a \diamond(b \diamond a)) \vee p_{l} \circ v_{P}(0) \\
& {[\text { by Proposition } 1] } \\
= & p_{l} \circ v_{P}(a \diamond(b \diamond a)) \text { for all } a, b \in A \\
& \text { and for } l=1,2, \ldots, m .
\end{aligned}
$$

(ii) $\Rightarrow$ (iii): It is known by Proposition 1 that $a \diamond(b \diamond a) \leqslant a$. Then by Proposition 3, $p_{l} \circ \mu_{P}(a) \leqslant p_{l} \circ \mu_{P}(a \diamond(b \diamond a)), p_{l} \circ \eta_{P}(a) \leqslant p_{l} \circ$ $\eta_{P}(a \diamond(b \diamond a))$ and $p_{l} \circ v_{P}(a) \geqslant p_{l} \circ v_{P}(a \diamond(b \diamond a))$ for all $a, b \in A$ and for $l=1,2, \ldots, m$. By (ii), $p_{l} \circ \mu_{P}(a) \geqslant p_{l} \circ \mu_{P}(a \diamond(b \diamond a)), p_{l} \circ \eta_{P}(a) \geqslant$ $p_{l} \circ \mu_{P}(a \diamond(b \diamond a))$ and $p_{l} \circ v_{P}(a) \leqslant p_{l} \circ v_{P}(a \diamond(b \diamond a))$ for all $a, b \in A$ and $l=1,2, \ldots, m$. As a result, $p_{l} \circ \mu_{P}(a)=p_{l} \circ \mu_{P}(a \diamond(b \diamond a))$, $p_{l} \circ \eta_{P}(a)=p_{l} \circ \mu_{P}(a \diamond(b \diamond a))$ and $p_{l} \circ v_{P}(a)=p_{l} \circ v_{P}(a \diamond(b \diamond a))$ for all $a, b \in A$ and for $l=1,2, \ldots, m$.

(iii) $\Rightarrow\left(\right.$ i): Since $P$ is an $m$-polar PFI of $A$ therefore $p_{l} \circ$ $\mu_{P}(a \diamond(b \diamond a)) \geqslant p_{l} \circ \mu_{P}\{a \diamond(b \diamond a) \diamond c\} \wedge p_{l} \circ \mu_{P}(c), p_{l} \circ$ $\eta_{P}(a \diamond(b \diamond a)) \geqslant p_{l} \circ \eta_{P}\{a \diamond(b \diamond a) \diamond c\} \wedge p_{l} \circ \eta_{P}(c)$ and $p_{l} \circ$ $v_{P}(a \diamond(b \diamond a)) \leqslant p_{l} \circ v_{P}\{a \diamond(b \diamond a) \diamond c\} \vee p_{l} \circ v_{P}(c)$ for all $a, b, c \in$ $A$ and for $l=1,2, \ldots, m$. By (iii), we have, $p_{l} \circ \mu_{P}(a) \geqslant p_{l} \circ$ $\mu_{P}\{a \diamond(b \diamond a) \diamond c\} \wedge p_{l} \circ \mu_{P}(c), p_{l} \circ \eta_{P}(a) \geqslant p_{l} \circ \eta_{P}\{a \diamond(b \diamond a) \diamond c\} \wedge$ $p_{l} \circ \eta_{P}(c)$ and $p_{l} \circ v_{P}(a) \leqslant p_{l} \circ v_{P}\{a \diamond(b \diamond a) \diamond c\} \vee p_{l} \circ v_{P}(c)$ for all $a, b, c \in A$ and for $l=1,2, \ldots, m$. Thus, $P$ is an $m$-polar PFII of $A$.

Definition 19. Let $P=\left(\mu_{P}, \eta_{P}, v_{P}\right)$ be an $m$-polar PFS in a BCK algebra $(A, \diamond, 0)$. Then $P$ is said to be an $m$-polar picture fuzzy positive implicative ideal (PFPII) if the below stated conditions are meet.

i. $\quad p_{l} \circ \eta_{P}(0) \geqslant p_{l} \circ \eta_{P}(a)$ and $p_{l} \circ v_{P}(0) \leqslant p_{l} \circ v_{P}(a)$

ii. $\quad p_{l} \circ \eta_{P}(a \diamond c) \geqslant p_{l} \circ \eta_{P}((a \diamond b) \diamond c) \wedge p_{l} \circ \eta_{P}(b \diamond c)$ and $p_{l} \circ$ $v_{P}(a \diamond c) \leqslant p_{l} \circ v_{P}((a \diamond b) \diamond c) \wedge p_{l} \circ v_{P}(b \diamond c), \forall a, b \in A$ and $l=1,2, \ldots, m$

Proposition 21. An m-polar PFI $P=\left(\mu_{P}, \eta_{P}, v_{P}\right)$ of a BCK algebra $(A, \diamond, 0)$ is an m-polar PFPII iff $p_{l} \circ \mu_{P}(a \diamond b) \geqslant p_{l} \circ \mu_{P}((a \diamond b) \diamond b)$, $p_{l} \circ \eta_{P}(a \diamond b) \geqslant p_{l} \circ \eta_{P}((a \diamond b) \diamond b)$ and $p_{l} \circ v_{P}(a \diamond b) \leqslant p_{l} \circ$ $v_{P}((a \diamond b) \diamond b), \forall a, b \in A$ and for $l=1,2, \ldots, m$.

Proof. The proof is easy. So, it is omitted here.

Since $(a \diamond b) \diamond b \leqslant a \diamond b$, it follows from Proposition 3 that $p_{l}$ 。 $\mu_{P}(a \diamond b) \leqslant p_{l} \circ \mu_{P}((a \diamond b) \diamond b), p_{l} \circ \eta_{P}(a \diamond b) \leqslant p_{l} \circ \eta_{P}((a \diamond b) \diamond b)$ and $p_{l} \circ v_{P}(a \diamond b) \geqslant p_{l} \circ v_{P}((a \diamond b) \diamond b), \forall a, b \in A$ and $l=1,2, \ldots, m$. So, the above Proposition can be modified in the following way:

Proposition 22. An m-polar PFI $P=\left(\mu_{P}, \eta_{P}, v_{P}\right)$ of a BCK algebra $(A, \diamond, 0)$ is a m-polar PFPII iff $p_{l} \circ \mu_{P}(a \diamond b)=p_{l} \circ \mu_{P}((a \diamond b) \diamond b)$, $p_{l} \circ \eta_{P}(a \diamond b)=p_{l} \circ \eta_{P}((a \diamond b) \diamond b)$ and $p_{l} \circ v_{P}(a \diamond b)=p_{l} \circ$ $v_{P}((a \diamond b) \diamond b), \forall a, b \in A$ and $l=1,2, \ldots, m$.

\section{7. m-POLAR PFCI}

Definition 20. Let $(A, \nabla, 0)$ be a BCK algebra and $P=\left(\mu_{P}, \eta_{P}, v_{P}\right)$ be an $m$-polar PFS in $A$. Then $P$ is said to be $m$-polar PFCI of $A$ if the following conditions are met:

i. $\quad p_{l} \circ \mu_{P}(0) \geqslant p_{l} \circ \mu_{P}(a), p_{l} \circ \eta_{P}(0) \geqslant p_{l} \circ \eta_{P}(a)$ and $p_{l} \circ v_{P}(0) \leqslant$ $p_{l} \circ v_{P}(a)$

ii. $\quad p_{l} \circ \mu_{P}(a \diamond(b \diamond(b \diamond a))) \geqslant p_{l} \circ \mu_{P}((a \diamond b) \diamond c) \wedge p_{l} \circ \mu_{P}(c), p_{l} \circ$ $\eta_{P}(a \diamond(b \diamond(b \diamond a))) \geqslant p_{l} \circ \eta_{P}((a \diamond b) \diamond c) \wedge p_{l} \circ \eta_{P}(c)$ and $p_{l} \circ$ $v_{P}(a \diamond(b \diamond(b \diamond a))) \leqslant p_{l} \circ v_{P}((a \diamond b) \diamond c) \vee p_{l} \circ v_{P}(c)$ for all $a, b \in A$ and for $l=1,2, \ldots, m$

Example 5. Let us consider the BCK algebra $(A, \diamond)$ as follows:

\begin{tabular}{lllll}
\hline$\diamond$ & 0 & $p$ & $q$ & $r$ \\
0 & 0 & 0 & 0 & 0 \\
$p$ & $p$ & 0 & 0 & $p$ \\
$q$ & $q$ & $p$ & 0 & $q$ \\
$r$ & $r$ & $r$ & $r$ & 0 \\
\hline
\end{tabular}

Now, let us suppose a 3-polar PFS $P=\left(\mu_{P}, \eta_{P}, v_{P}\right)$ defined by

$$
\begin{gathered}
\mu_{P}(a)= \begin{cases}(0.34,0.36,0.37), & \text { if } a=0 \\
(0.28,0.3,0.32), & \text { if } a=p \\
(0.17,0.18,0.18), & \text { if } a=q, r\end{cases} \\
\eta_{P}(a)= \begin{cases}(0.35,0.36,0.39), & \text { if } a=0 \\
(0.25,0.27,0.3), & \text { if } a=p \\
(0.2,0.23,0.27), & \text { if } a=q, r\end{cases}
\end{gathered}
$$

and

$$
\eta_{P}(a)= \begin{cases}(0.1,0.15,0.17), & \text { if } a=0 \\ (0.2,0.27,0.31), & \text { if } a=p \\ (0.55,0.57,0.58), & \text { if } a=q, r\end{cases}
$$

Clearly, $P$ is a 3-polar PFCI of $A$.

Definition 21. A BCK algebra $(A, \diamond, 0)$ is said to be commutative if $b \diamond(b \diamond a)=a \diamond(a \diamond b)$ for all $a, b \in A$.

Proposition 23. Every m-polar PFCI of a BCK algebra is an m-polar PFI.

Proof. Let $P=\left(\mu_{P}, \eta_{P}, v_{P}\right)$ is an $m$-polar PFCI of a BCK algebra $(A, \diamond, 0)$.

Now, $(a \diamond(0 \diamond(0 \diamond a)))$

$=(a \diamond 0)[$ by Proposition 1]

$=a[$ by Proposition 1]

Now, $p_{l} \circ \mu_{P}(a)=p_{l} \circ \mu_{P}(a \diamond(0 \diamond(0 \diamond a))) \geqslant p_{l} \circ \mu_{P}((a \diamond 0) \diamond c) \wedge$ $p_{l} \circ \mu_{P}(c)=p_{l} \circ \mu_{P}(a \diamond c) \wedge p_{l} \circ \mu_{P}(c), p_{l} \circ \eta_{P}(a)=p_{l} \circ$ $\eta_{P}(a \diamond(0 \diamond(0 \diamond a))) \geqslant p_{l} \circ \eta_{P}((a \diamond 0) \diamond c) \wedge p_{l} \circ \eta_{P}(c)=p_{l} \circ$ $\eta_{P}(a \diamond c) \wedge p_{l} \circ \eta_{P}(c)$ and $p_{l} \circ v_{P}(a)=p_{l} \circ v_{P}(a \diamond(0 \diamond(0 \diamond a))) \leqslant$ $p_{l} \circ v_{P}((a \diamond 0) \diamond c) \vee p_{l} \circ v_{P}(c)=p_{l} \circ v_{P}(a \diamond c) \vee p_{l} \circ v_{P}(c)$ for all $a, c \in A$ and for $l=1,2, \ldots, m$. Consequently, $P$ is an $m$-polar PFI of $A$. 
The above proposition is not true in reverse direction which is clear from following example. But the converse of the above proposition holds in commutative BCK algebra which is highlighted through Proposition 24.

Example 6. Let us consider a BCK algebra $(A, \diamond)$ as follows:

\begin{tabular}{llllll}
\hline$\diamond$ & 0 & $p$ & $q$ & $r$ & $s$ \\
0 & 0 & 0 & 0 & 0 & 0 \\
$p$ & $p$ & 0 & $p$ & 0 & 0 \\
$q$ & $q$ & $q$ & 0 & 0 & 0 \\
$r$ & $r$ & $r$ & $r$ & 0 & 0 \\
$s$ & $s$ & $s$ & $s$ & $r$ & 0 \\
\hline
\end{tabular}

Now, let us suppose a 3-polar PFS $P=\left(\mu_{P}, \eta_{P}, v_{P}\right)$ defined by

$$
\begin{gathered}
\mu_{P}(a)= \begin{cases}(0.4,0.41,0.43), & \text { if } a=0 \\
(0.3,0.32,0.33), & \text { if } a=p \\
(0.2,0.24,0.27), & \text { if } a=q, r, s\end{cases} \\
\eta_{P}(a)= \begin{cases}(0.43,0.45,0.47), & \text { if } a=0 \\
(0.35,0.36,0.37), & \text { if } a=p \\
(0.21,0.22,0.23), & \text { if } a=q, r, s\end{cases} \\
v_{P}(a)= \begin{cases}(0.08,0.09,0.1), & \text { if } a=0 \\
(0.27,0.28,0.3), & \text { if } a=p \\
(0.45,0.47,0.5), & \text { if } a=q, r, s\end{cases}
\end{gathered}
$$

Clearly, $P$ is a 3-polar PFI of $A$.

It is observed that

$$
\begin{aligned}
& \mu_{P}((q \diamond(r \diamond(r \diamond q))))=\mu_{P}(q)=(0.2,0.24,0.27), \\
& \mu_{P}((q \diamond r) \diamond 0) \wedge \mu_{P}(0)=(0.4,0.41,0.43) \\
& \eta_{P}((q \diamond(r \diamond(r \diamond q))))=\eta_{P}(q)=(0.21,0.22,0.23), \\
& \eta_{P}((q \diamond r) \diamond 0) \wedge \eta_{P}(0)=(0.43,0.45,0.47) \\
& v_{P}((q \diamond(r \diamond(r \diamond q))))=v_{P}(q)=(0.45,0.47,0.5), \\
& v_{P}((q \diamond r) \diamond 0) \vee v_{P}(0)=(0.08,0.09,0.1) .
\end{aligned}
$$

Here, $\mu_{P}((q \diamond(r \diamond(r \diamond q)))) \ngtr \mu_{P}((q \diamond r) \diamond 0) \wedge \mu_{P}(0), \eta_{P}((q \diamond(r \diamond$ $(r \diamond q)))) \not \eta_{P}((q \diamond r) \diamond 0) \wedge \eta_{P}(0)$ and $v_{P}((q \diamond(r \diamond(r \diamond q)))) \quad \not \quad$ $v_{P}((q \diamond r) \diamond 0) \vee v_{P}(0)$. Clearly, $P$ is not a 3-polar PFCI of $A$.

Proposition 24. In a commutative BCK algebra, every m-polar PFI is an m-polar PFCI.

Proof. Let $P=\left(\mu_{P}, \eta_{P}, v_{P}\right)$ be an $m$-polar PFI of a commutative BCK algebra $(A, \diamond, 0)$. We have, $[((\mathrm{a} \diamond(b \diamond(b \diamond a))) \diamond((a \diamond b)$ $\diamond c))] \diamond c=((a \diamond(b \diamond(b \diamond a))) \diamond c) \diamond((a \diamond b) \diamond c)$ [by Proposition 1]

$\leqslant(a \diamond(b \diamond(b \diamond a))) \diamond(a \diamond b)$ [by Proposition 1]

$=(a \diamond(a \diamond(a \diamond b))) \diamond(a \diamond b)$ [as $A$ is commutative therefore $(a \diamond(a \diamond b))=(b \diamond(b \diamond a))$ for all $a, b \in A]$

$=(a \diamond b) \diamond(a \diamond b)$ [by Proposition 1]

$=0$

i.e. $(a \diamond(b \diamond(b \diamond a))) \diamond((a \diamond b) \diamond c) \leqslant c$.
Thus, by Proposition 4, it is obtained that $p_{l}$ 。 $\mu_{P}((a \diamond(b \diamond(b \diamond a)))) \geqslant p_{l} \circ \mu_{P}(((a \diamond b) \diamond c)) \wedge p_{l} \circ \mu_{P}(c)$, $p_{l} \circ \eta_{P}((a \diamond(b \diamond(b \diamond a)))) \geqslant p_{l} \circ \eta_{P}(((a \diamond b) \diamond c)) \wedge p_{l} \circ \eta_{P}(c)$ and $p_{l} \circ v_{P}((a \diamond(b \diamond(b \diamond a)))) \leqslant p_{l} \circ v_{P}(((a \diamond b) \diamond c)) \vee p_{l} \circ v_{P}(c)$ for all $a, b, c \in A$ and for $l=1,2, \ldots, m$. Consequently, $P$ is an $m$-polar PFCI of $A$.

Now, we are interested to develop a relationship between $m$-polar PFII and $m$-polar PFCI. Before that we state some propositions which are necessary in this regard.

Meng et al. [10] stated the following proposition:

Proposition 25. The followings hold in a BCK algebra $(A, \diamond, 0)$.

$$
\begin{aligned}
\text { i. } & ((a \diamond c) \diamond c) \diamond(b \diamond c) \leqslant(a \diamond b) \diamond c . \\
\text { ii. } & (a \diamond c) \diamond(a \diamond(a \diamond c))=(a \diamond c) \diamond c . \\
\text { iii. } & (a \diamond(b \diamond(b \diamond a))) \diamond(b \diamond(a \diamond(b \diamond(b \diamond a)))) \leqslant a \diamond b .
\end{aligned}
$$

Proposition 26. An m-polar PFI $P=\left(\mu_{P}, \eta_{P}, v_{P}\right)$ of a BCK algebra $(A, \diamond, 0)$ is an m-polar PFCI iff $p_{l} \circ \mu_{P}(a \diamond(b \diamond(b \diamond a))) \geqslant$ $p_{l} \circ \mu_{P}(a \diamond b), p_{l} \circ \eta_{P}(a \diamond(b \diamond(b \diamond a))) \geqslant p_{l} \circ \eta_{P}(a \diamond b)$ and $p_{l} \circ$ $v_{P}(a \diamond(b \diamond(b \diamond a))) \leqslant p_{l} \circ v_{P}(a \diamond b), \forall a, b \in A$ and $l=1,2, \ldots, m$.

Proof. The proof is easy. So, it is omitted here.

It is observed that $a \diamond b \leqslant a \diamond(b \diamond(b \diamond a))$ and using Proposition 3 we get, $p_{l} \circ \mu_{P}(a \diamond(b \diamond(b \diamond a))) \leqslant p_{l} \circ \mu_{P}(a \diamond b), p_{l} \circ$ $\eta_{P}(a \diamond(b \diamond(b \diamond a))) \leqslant p_{l} \circ \eta_{P}(a \diamond b)$ and $p_{l} \circ v_{P}(a \diamond(b \diamond(b \diamond a))) \geqslant$ $p_{l} \circ v_{P}(a \diamond b), \forall a, b \in A$ and $l=1,2, \ldots, m$. So, above Proposition can be modified in the following way:

Proposition 27. An m-polar PFI $P=\left(\mu_{P}, \eta_{P}, v_{P}\right)$ of a BCK algebra $(A, \diamond, 0)$ is an m-polar PFCI iff $p_{l} \circ \mu_{P}(a \diamond(b \diamond(b \diamond a)))=$ $p_{l} \circ \mu_{P}(a \diamond b), p_{l} \circ \eta_{P}(a \diamond(b \diamond(b \diamond a)))=p_{l} \circ \eta_{P}(a \diamond b)$ and $p_{l} \circ$ $v_{P}(a \diamond(b \diamond(b \diamond a)))=p_{l} \circ v_{P}(a \diamond b), \forall a, b \in A$ and $l=1,2, \ldots, m$.

Proposition 28. An m-polar PFI P $=\left(\mu_{P}, \eta_{P}, v_{P}\right)$ is an m-polar PFII iff $P$ is both $m$-polar PFCI and $m$-polar PFPII.

Proof. Suppose that $P$ is $m$-polar PFII. Then by Proposition 25 (i) and Proposition 4,

$$
\begin{aligned}
& p_{l} \circ \mu_{P}((a \diamond b) \diamond c) \wedge p_{l} \circ \mu_{P}(b \diamond c) \\
& \leqslant p_{l} \circ \mu_{P}((a \diamond c) \diamond c) \\
& =p_{l} \circ \mu_{P}((a \diamond c) \diamond(a \diamond(a \diamond c))) \text { [by Proposition } 25 \text { (ii)] } \\
& =p_{l} \circ \mu_{P}(a \diamond c)[\text { by Proposition } 20 \text { (iii)], } \\
& p_{l} \circ \eta_{P}((a \diamond b) \diamond c) \wedge p_{l} \circ \eta_{P}(b \diamond c) \\
& \leqslant p_{l} \circ \eta_{P}((a \diamond c) \diamond c) \\
& =p_{l} \circ \eta_{P}((a \diamond c) \diamond(a \diamond(a \diamond c))) \text { [by Proposition } 25 \text { (ii)] } \\
& =p_{l} \circ \eta_{P}(a \diamond c)[\text { by Proposition } 20 \text { (iii)] } \\
& \text { and } p_{l} \circ v_{P}((a \diamond b) \diamond c) \wedge p_{l} \circ v_{P}(b \diamond c) \\
& \geqslant p_{l} \circ v_{P}((a \diamond c) \diamond c) \\
& =p_{l} \circ v_{P}((a \diamond c) \diamond(a \diamond(a \diamond c))) \text { [by Proposition } 25 \text { (ii)] } \\
& =p_{l} \circ v_{P}(a \diamond c)[\text { by Proposition 20 (iii)] }
\end{aligned}
$$

Therefore, $P$ is an $m$-polar PFPII. 
By Proposition 25 (iii) and Proposition 3 we get,

$$
\begin{aligned}
& p_{l} \circ \mu_{P}(a \diamond b) \\
& \left.\leqslant p_{l} \circ \mu_{P}(a \diamond(b \diamond(b \diamond a))) \diamond(b \diamond(a \diamond(b \diamond(b \diamond a))))\right) \\
& =p_{l} \circ \mu_{P}((a \diamond(b \diamond(b \diamond a)))[\text { by Proposition } 20 \text { (iii)], } \\
& p_{l} \circ \eta_{P}(a \diamond b) \\
& \left.\leqslant p_{l} \circ \eta_{P}(a \diamond(b \diamond(b \diamond a))) \diamond(b \diamond(a \diamond(b \diamond(b \diamond a))))\right) \\
& =p_{l} \circ \eta_{P}((a \diamond(b \diamond(b \diamond a)))[\text { by Proposition } 20 \text { (iii)] } \\
& \text { and } p_{l} \circ v_{P}(a \diamond b) \\
& \left.\geqslant p_{l} \circ v_{P}(a \diamond(b \diamond(b \diamond a))) \diamond(b \diamond(a \diamond(b \diamond(b \diamond a))))\right) \\
& =p_{l} \circ v_{P}((a \diamond(b \diamond(b \diamond a)))[\text { by Proposition } 20 \text { (iii)]. }
\end{aligned}
$$

Therefore, $P$ is an $m$-polar PFCI of $A$.

Conversely, let $P$ be both $m$-polar PFPII and $m$-polar PFCI of $A$. Since $(b \diamond(b \diamond a)) \diamond(b \diamond a) \leqslant a \diamond(b \diamond a)$, by Proposition 3,

$$
\begin{aligned}
& p_{l} \circ \mu_{P}(a \diamond(b \diamond a)) \\
& \leqslant p_{l} \circ \mu_{P}((b \diamond(b \diamond a)) \diamond(b \diamond a)), \\
& p_{l} \circ \eta_{P}(a \diamond(b \diamond a)) \\
& \leqslant p_{l} \circ \eta_{P}((b \diamond(b \diamond a)) \diamond(b \diamond a)) \\
& \text { and } p_{l} \circ v_{P}(a \diamond(b \diamond a)) \\
& \geqslant p_{l} \circ v_{P}((b \diamond(b \diamond a)) \diamond(b \diamond a))
\end{aligned}
$$

By Proposition 22,

$$
\begin{aligned}
p_{l} \circ \mu_{P}((b \diamond(b \diamond a)) \diamond(b \diamond a)) & =p_{l} \circ \mu_{P}(b \diamond(b \diamond a)), \\
p_{l} \circ \eta_{P}((b \diamond(b \diamond a)) \diamond(b \diamond a)) & =p_{l} \circ \eta_{P}(b \diamond(b \diamond a)) \\
\text { and } p_{l} \circ v_{P}((b \diamond(b \diamond a)) \diamond(b \diamond a)) & =p_{l} \circ v_{P}(b \diamond(b \diamond a))
\end{aligned}
$$

therefore it is obtained that

$$
\begin{gathered}
p_{l} \circ \mu_{P}(a \diamond(b \diamond a)) \leqslant p_{l} \circ \mu_{P}(b \diamond(b \diamond a)), \\
p_{l} \circ \eta_{P}(a \diamond(b \diamond a)) \leqslant p_{l} \circ \eta_{P}(b \diamond(b \diamond a)) \\
\text { and } p_{l} \circ v_{P}(a \diamond(b \diamond a)) \geqslant p_{l} \circ v_{P}(b \diamond(b \diamond a))
\end{gathered}
$$

Also, $a \diamond b \leqslant a \diamond(b \diamond a)$. Therefore, by Proposition 3,

$$
\begin{aligned}
p_{l} \circ \mu_{P}(a \diamond(b \diamond a)) & \leqslant p_{l} \circ \mu_{P}(a \diamond b), \\
p_{l} \circ \eta_{P}(a \diamond(b \diamond a)) & \leqslant p_{l} \circ \eta_{P}(a \diamond b) \\
\text { and } p_{l} \circ v_{P}(a \diamond(b \diamond a)) & \geqslant p_{l} \circ v_{P}(a \diamond b)
\end{aligned}
$$

Since $P$ is an $m$-polar PFCI therefore by Proposition 27,

$$
\begin{aligned}
p_{l} \circ \mu_{P}(a \diamond b) & =p_{l} \circ \mu_{P}(a \diamond(b \diamond(b \diamond a))), \\
p_{l} \circ \eta_{P}(a \diamond b) & =p_{l} \circ \eta_{P}(a \diamond(b \diamond(b \diamond a))) \\
\text { and } p_{l} \circ v_{P}(a \diamond b) & =p_{l} \circ v_{P}(a \diamond(b \diamond(b \diamond a)))
\end{aligned}
$$

Hence it is obtained that

$$
\begin{gathered}
p_{l} \circ \mu_{P}(a \diamond(b \diamond a)) \leqslant p_{l} \circ \mu_{P}(a \diamond(b \diamond(b \diamond a))), \\
p_{l} \circ \eta_{P}(a \diamond(b \diamond a)) \leqslant p_{l} \circ \eta_{P}(a \diamond(b \diamond(b \diamond a))) \\
\text { and } p_{l} \circ v_{P}(a \diamond(b \diamond a)) \geqslant p_{l} \circ v_{P}(a \diamond(b \diamond(b \diamond a)))
\end{gathered}
$$

Combining (1) and (4), (2) and (5), (3) and (6) it is obtained that

$$
\begin{aligned}
& p_{l} \circ \mu_{P}(a \diamond(b \diamond a)) \\
& \leqslant p_{l} \circ \mu_{P}(a \diamond(b \diamond(b \diamond a))) \wedge p_{l} \circ \mu_{P}(b \diamond(b \diamond a)) \\
& \leqslant p_{l} \circ \mu_{P}(a), \\
& p_{l} \circ \eta_{P}(a \diamond(b \diamond a)) \\
& \leqslant p_{l} \circ \eta_{P}(a \diamond(b \diamond(b \diamond a))) \wedge p_{l} \circ \eta_{P}(b \diamond(b \diamond a)) \\
& \leqslant p_{l} \circ \eta_{P}(a) \\
& \text { and } p_{l} \circ v_{P}(a \diamond(b \diamond a)) \\
& \geqslant p_{l} \circ v_{P}(a \diamond(b \diamond(b \diamond a))) \vee p_{l} \circ v_{P}(b \diamond(b \diamond a)) \\
& \geqslant p_{l} \circ v_{P}(a) .
\end{aligned}
$$

So, by Proposition 20 (ii), $P$ is an $m$-polar PFII of $A$.

\section{CONCLUSION}

In this paper, we have initiated the notion of $m$-polar PFI and $m$ polar PFII of BCK algebra. We have studied some basic results related to them. We have established a relationship between $m$-polar PFI and $m$-polar PFII of a BCK algebra. We have also investigated a relationship between $m$-polar PFI and $m$-polar PFCI. We have studied some properties of $m$-polar PFI under homomorphism of $\mathrm{BCK}$ algebra. It is our hope that our works will help the researchers to study some other types of algebraic structures in context of $m$-polar PFS.

\section{CONFLICT OF INTEREST}

Authors declare that they have no conflict of interest.

\section{AUTHORS' CONTRIBUTIONS}

S. Dogra Writing, reviewing and editing. M. Pal reviewing, editing and supervision.

\section{Funding Statement}

There is no funding source for this work.

\section{ACKNOWLEDGMENTS}

Authors are thankful to the reviewers for their valuable suggestions towards the improvement of the paper.

\section{REFERENCES}

[1] L.A. Zadeh, Fuzzy sets, Inf. Control. 8 (1965), 338-353.

[2] K.T. Atanassov, Intuitionistic fuzzy sets, Fuzzy Sets Syst. 20 (1986), 87-96.

[3] Y. Imai, K. Iseki, On axiom systems of propositional calculi XIV, Proc. Japan Acad. 42 (1966), 19-22.

[4] K. Iseki, S. Tanaka, An introduction to the theory of BCK algebras, Math. Japonica. 23 (1978), 1-26.

[5] K. Iseki, On BCI-algebras, Math. Semin. Notes. 8 (1980), 125-130.

[6] O.G. Xi, Fuzzy BCK algebras, Math. Japonica. 36 (1991), 935-942.

[7] B. Ahmad, Fuzzy BCI algebras, J. Fuzzy Math. 1 (1993), 445-452. 
[8] Y.B. Jun, Closed fuzzy ideals in BCI algebras, Math. Japonica. 38 (1993), 199-202.

[9] Y.B. Jun, E.H. Roh, Fuzzy commutative ideals of BCK algebra, Fuzzy Sets Syst. 64 (1994), 401-405.

[10] J. Meng, Y.B. Jun, H.S. Kim, Fuzzy implicative ideals of BCKalgebras, Fuzzy Sets Syst. 89 (1997), 243-248.

[11] A.B. Saeid, Fuzzy dot BCK/BCI algebras, Int. J. Algebra. 4 (2012), 341-352.

[12] T. Senapati, M. Bhowmik, M. Pal, B. Davvaz, Fuzzy translations of fuzzy H-ideals in BCK/BCI algebras, Indones. Math. Soc. 21 (2015), 45-58.

[13] Y.B. Jun, K.H. Kim, Intuitionistic fuzzy ideals of BCK-algebras, Int. J. Math. Math. Sci. 24 (2000), 839-849.

[14] T. Senapati, M. Bhowmik, M. Pal, Atanassov intuitionistic fuzzy translations of intuitionistic fuzzy $\mathrm{H}$-ideals in BCK/BCI algebras, Notes Intuitionistic Fuzzy Sets. 19 (2013), 32-47.

[15] T. Senapati, M. Bhowmik, M. Pal, B. Davvaz, Atanassov's intuitionistic fuzzy translations of intuitionistic fuzzy subalgebras and ideals in BCK/BCI algebras, Eurasian Math. J. 6 (2015), 96-114.
[16] W.R. Zhang, Bipolar fuzzy sets and relations: a computational framework for cognitive modeling and multiagent decision analysis, in Proceedings of the Industrial Fuzzy Control and Intelligent Systems Conference, and the NASA Joint Technology Workshop on Neural Networks and Fuzzy Logic and Fuzzy Information Processing Society Biannual Conference, San Antonio, TX, USA, 1994, pp. 305-309.

[17] J. Chen, et al., m-Polar fuzzy sets: an extension of bipolar fuzzy sets, Sci. World J. 2014 (2014), 1-8.

[18] B.C. Cuong, V. Kreinovich, Picture fuzzy sets - a new concept for computational intelligence problems, in Proceedings of the Third World Congress on Information and Communication Technologies WIICT, Hanoi, Vietnam, 2013.

[19] B.C. Cuong, Picture fuzzy sets, J. Comput. Sci. Cybern. 30 (2014), 409-420.

[20] B.C. Cuong, P.V. Hai, Some fuzzy logic operators for picture fuzzy sets, in Seventh International Conference on Knowledge and Systems Engineering, Ho Chi Minh City, Vietnam, 2015.

[21] P. Dutta, S. Ganju, Some aspects of picture fuzzy set, Trans. A. Razmandze Math. Inst. 172 (2018), 164-175. 